\title{
Self- and Zinc Diffusion in Gallium Antimonide
}

\author{
by \\ Samuel Piers Nicols \\ B.S. (University of California, San Diego) 1998 \\ A thesis submitted in partial satisfaction of the \\ requirements for the degree of \\ Masters of Science \\ in \\ Engineering - Materials Science \& Engineering \\ in the \\ GRADUATE DIVISION \\ of the \\ UNIVERSITY OF CALIFORNIA, BERKELEY
}

committee in charge:

Professor Eugene E. Haller, Chair

Professor Hartmut A. Bracht

Professor Eicke R. Weber

Spring 2002 


\section{Acknowledgements}

I would like to thank a number of people who were instrumental in completing this thesis. First, I would like to thank Prof. Haller for giving me the opportunity to tackle this diffusion project. He was always ready to provide advice, support, and direction without hesitation. I especially appreciate the trust that he gave me in nearly all aspects of my research, particularly in preparing for and attending the $21^{\text {st }}$ International Conference on Defects in Semiconductors in Germany. A very special thanks must also go to Prof. Hartmut Bracht at the University of Münster who in his collaboration provided the diffusion expertise to develop, model, and explore the results of this project. Even with the long distance separating us, I could always count on Prof. Bracht to immediately respond to issues arising in any aspect of the research. I would like to thank Jeff Beeman for discussions not only concerning experimental setups, but also about the questions in life. David Hom was also instrumental in setting me up at the lab, as well as taking the time to answer any and all the questions I had. I would also like to thank all of the graduate students on the second floor of building two at Lawrence Berkeley National Laboratory for their camaraderie and the cutthroat card games, particularly Abraham Anapolsky, Ben Cardozo and Jordana Bandaru. Finally I would like to thank my family for their continued support through my entire education, and particularly to Anna for reminding me how important it is to be silly sometimes.

This work was supported in part by the Director, Office of Science, Office of Basic Energy Sciences, Materials Sciences Division of the U.S. Department of Energy under Contract No. DE-AC03-76SF00098 and by U.S. NSF Grant Nos. DMR-97 32707 and DMR-01 09644. 


\section{Table of Contents}

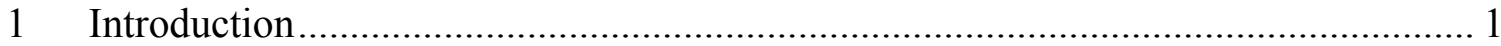

$1.1 \quad$ Key Physical Properties of GaSb ............................................................. 2

1.2 Crystal Structure and Point Defects............................................................. 4

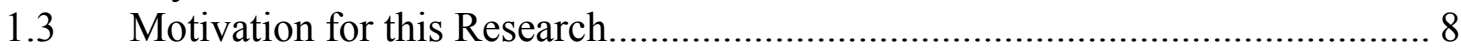

$1.4 \quad$ Previous Diffusion Studies ........................................................................ 10

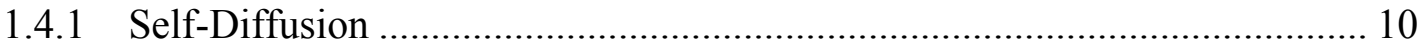

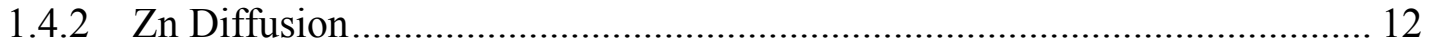

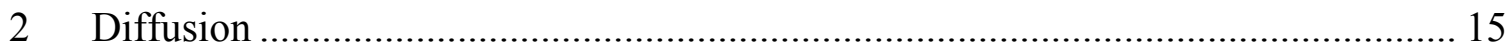

$2.1 \quad$ Development of Diffusion Theory............................................................. 15

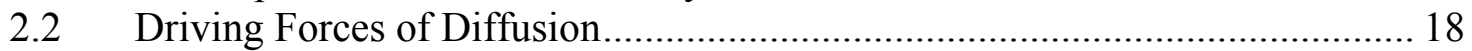

2.3 Microscopic Mechanisms of Diffusion.......................................................... 20

2.4 Experimental Methods for Measuring Diffusion.............................................. 25

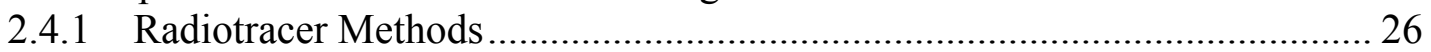

2.4.2 Isotopically Enriched Samples............................................................... 27

2.4.3 Closed Ampoule Annealing ................................................................... 30

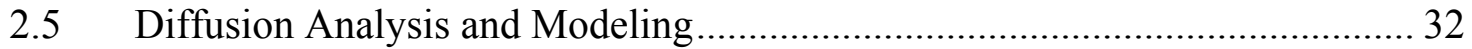

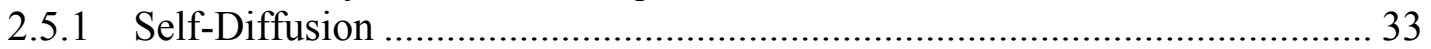

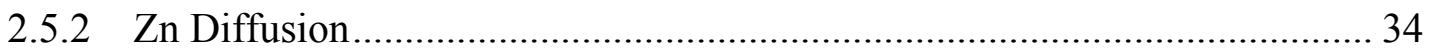

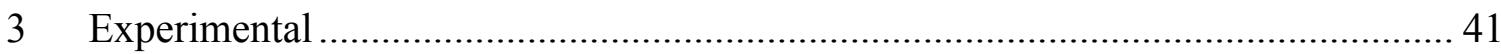

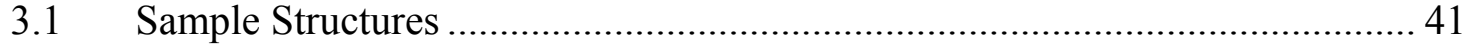

3.1.1 Isotopic Heterostructures …………………........................................... 41

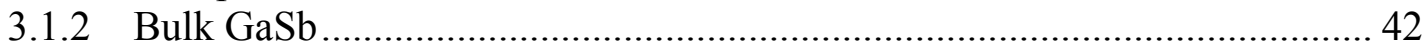

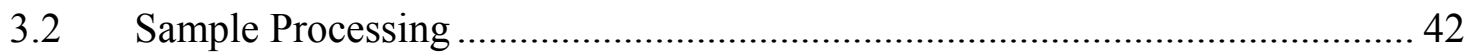

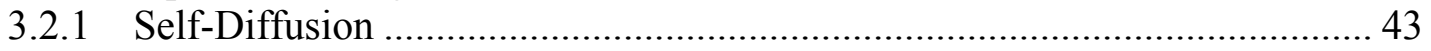

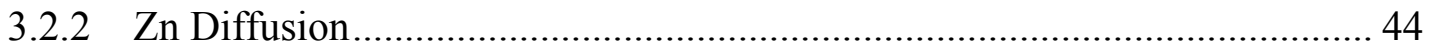

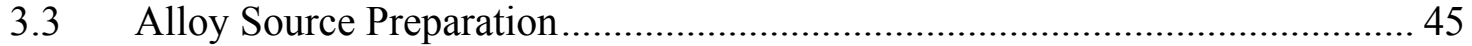

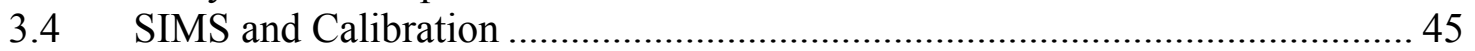

3.5 Transmission Electron Microscopy ............................................................... 46

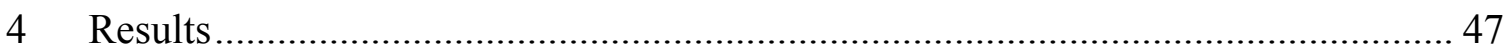

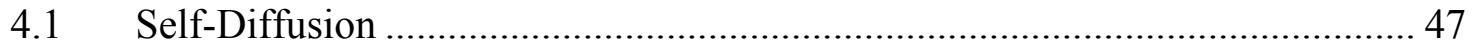

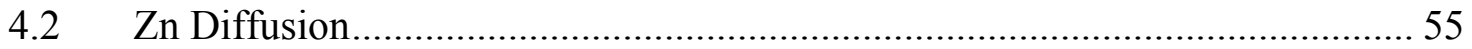

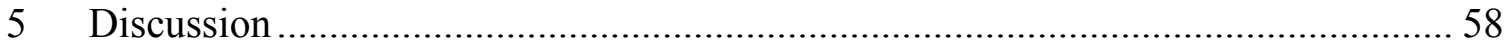

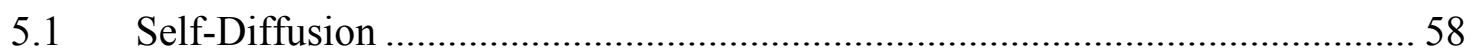

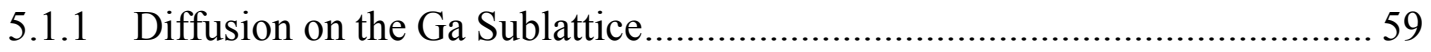

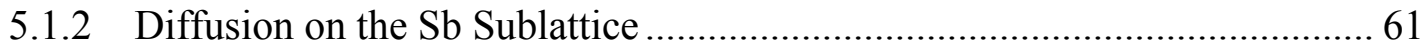

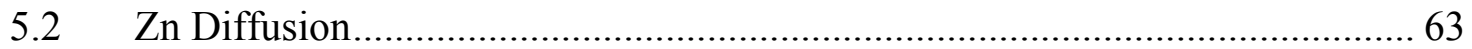

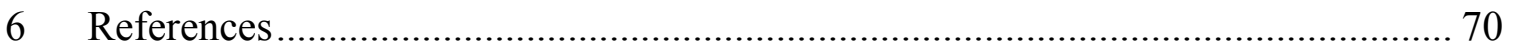

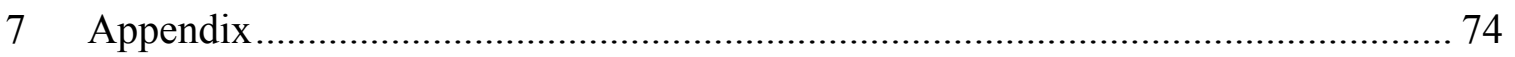

7.1 Secondary Ion Mass Spectrometry (SIMS) ………….................................. 74

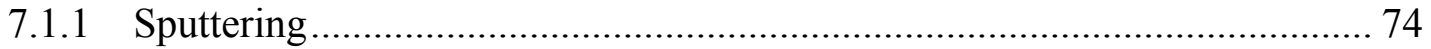

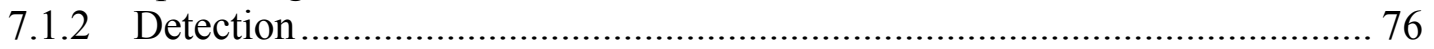




\section{Introduction}

The technological age has in large part been driven by the applications of semiconductors, and most noteably by silicon. Our lives have been thoroughly changed by devices using the broad range of semiconductor technology developed over the past forty years. Much of the technological development has its foundation in research carried out on the different semiconductors whose properties can be exploited to make transistors, lasers, and many other devices. While the technological focus has largely been on silicon, many other semiconductor systems have applications in industry and offer formidable academic challenges.

Diffusion studies belong to the most basic studies in semiconductors, important from both an application as well as research standpoint. Diffusion processes govern the junctions formed for device applications. As the device dimensions are decreased and the dopant concentrations increased, keeping pace with Moore's Law, a deeper understanding of diffusion is necessary to establish and maintain the sharp dopant profiles engineered for optimal device performance. From an academic viewpoint, diffusion in semiconductors allows for the study of point defects. Very few techniques exist which allow for the extraction of as much information of their properties.

This study focuses on diffusion in the semiconductor gallium antimonide ( $\mathrm{GaSb})$. As will become clear, this compound semiconductor proves to be a powerful one for investigating both self- and foreign atom diffusion. While the results have direct applications for work on GaSb devices, the results should also be taken in the broader context of III-V semiconductors. Results here can be compared and contrasted to results 
in systems such as GaAs and even GaN, indicating trends within this common group of semiconductors. The results also have direct importance for ternary and quaternary semiconductor systems used in devices such as high speed $\mathrm{InP} / \mathrm{GaAsSb} / \mathrm{InP}$ double heterojunction bipolar transistors (DHBT) [Dvorak, (2001)].

Many of the findings which will be reported here were previously published in three journal articles. Hartmut Bracht was the lead author on two articles on selfdiffusion studies in GaSb [Bracht, (2001), (2000)], while this report's author was the lead author on Zn diffusion results [Nicols, (2001)]. Much of the information contained herein can be found in those articles, but a more detailed treatment is presented here.

\subsection{Key Physical Properties of GaSb}

$\mathrm{GaSb}$ is a III-V type semiconductor with a direct band gap of $0.725 \mathrm{eV}$ at room temperature. It crystallizes in the zinc-blende structure with a lattice parameter of 6.096 $\AA$ at the melting point of $712^{\circ} \mathrm{C}$. GaSb can be readily obtained in bulk form through the Czochralski $(\mathrm{Cz})$ growth technique. The phase diagram for $\mathrm{GaSb}$ has been extensively investigated, and is shown in Figure 1-1. Due to the relatively high vapor pressure of $\mathrm{Sb}$ compared to $\mathrm{Ga}$ at high temperatures, $\mathrm{GaSb}$ is usually grown with a slight excess of $\mathrm{Sb}$ to compensate for the small amount that volatilizes. 


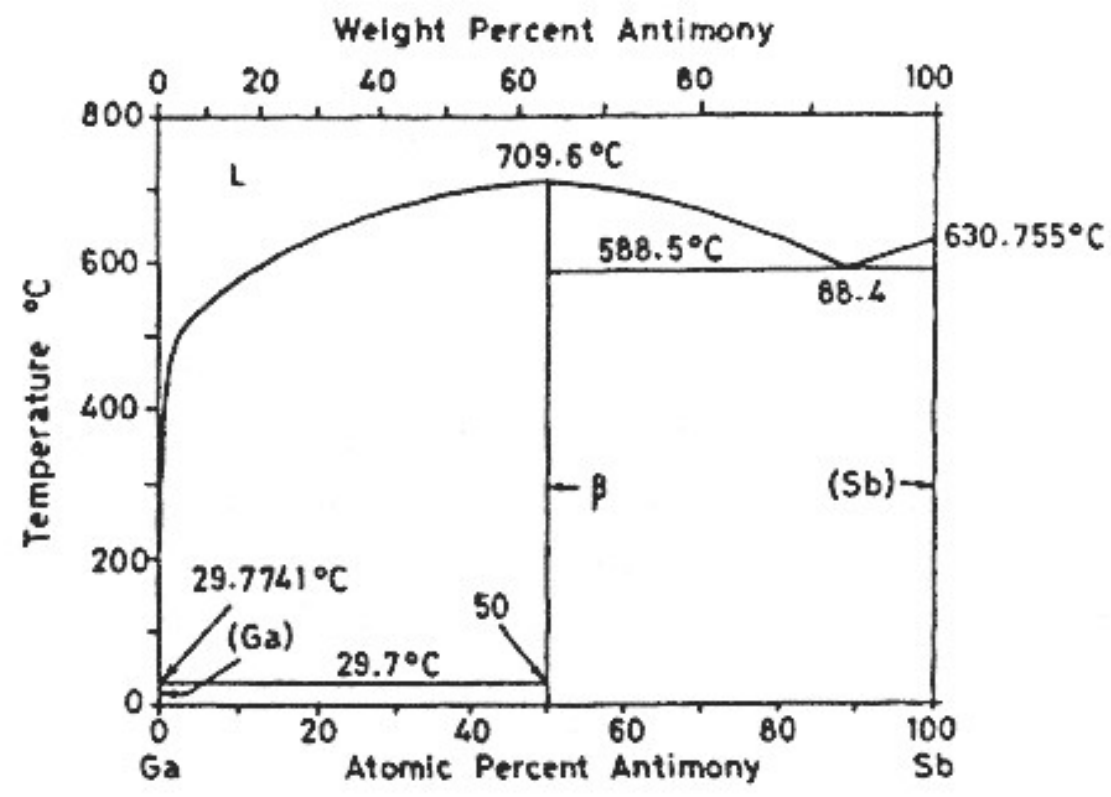

Figure 1-1: Phase diagram of GaSb [Hansen, (1958)]. The melting point was later adjusted to $712^{\circ} \mathrm{C}$.

The thermo-physical properties of $\mathrm{GaSb}$ are well suited to the growth of low dislocation density crystals. Crystals are usually grown in an oxygen-free hydrogen ambient to limit the formation of $\mathrm{Ga}_{2} \mathrm{O}_{3}$ on the melt surface which promotes twinning and impairs seeding [McAfee, (1986)]. 2-inch diameter crystals can be grown with the current technology.

Instrinsic GaSb, including GaSb grown via the Czochralski technique, has been shown to have a residual p-type conductivity. The responsible acceptors have been attributed to a native defect of acceptor type since chemical impurities could be ruled out on the basis of concentration already in the early 60's. Many experiments were carried out at that time to determine the nature of the defect. Ion pairing studies using Li were successful in neutralizing the residual acceptors [Baxter, (1965]. Growth from nonstoichiometric melts also provided some insight into the nature of the defect. A significant reduction of the background acceptor concentration is seen in crystals grown 
from melts with greater than $60 \% \mathrm{Sb}$ concentration [Reid, (1966)]. This would point to the defect being related to either an excess of Ga or a deficiency of Sb within the crystalline phase. The most widely accepted model suggests that the native defect responsible for the residual p-type behavior is a $\mathrm{Ga}$ vacancy $-\mathrm{Ga}$ antisite $\left(\mathrm{V}_{\mathrm{Ga}} \mathrm{Ga}_{\mathrm{Sb}}\right)$ double-defect, proposed first by Reid and collaborators [Reid, (1966)]. This model is supported by thermodynamic investigations [Ichimura, (1990)]. The double defect was called into question after it was found to have tetrahedral electronic symmetry through Zeeman experiments [Ruhle, (1975)]. The defects' physical structure, however, does not necessarily have to correlate to the electronic structure.

\subsection{Crystal Structure and Point Defects}

GaSb crystallizes in the zinc-blende structure, whose unit cell is represented in Figure 1-2. Figure 1-3 offers a more useful visualization of the structure, the $4 \times 4$ representation. The zinc-blende structure is made of up two interlocked face-centered cubic (FCC) lattices. In a perfect crystal, each atom of one species is surrounded by 4 atoms of the other species in a tetrahedral arrangement. The zinc-blende structure has the distinct property that it is a very open lattice, leaving space for relatively large interstitial species. In GaSb, where the covalent radii of $\mathrm{Ga}$ and $\mathrm{Sb}$ are 1.26 and $1.38 \AA$ respectively, $66 \%$ of the lattice volume is empty. 


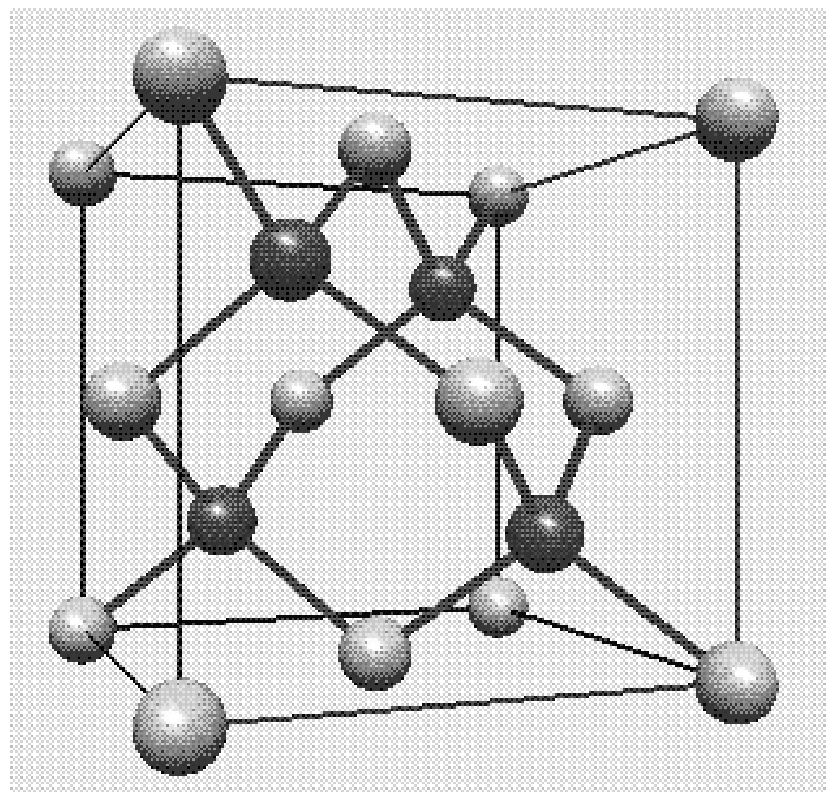

Figure 1-2: Zinc-blende structure of any III-V compound semicondctor, including GaSb [Akopian, (2000)]

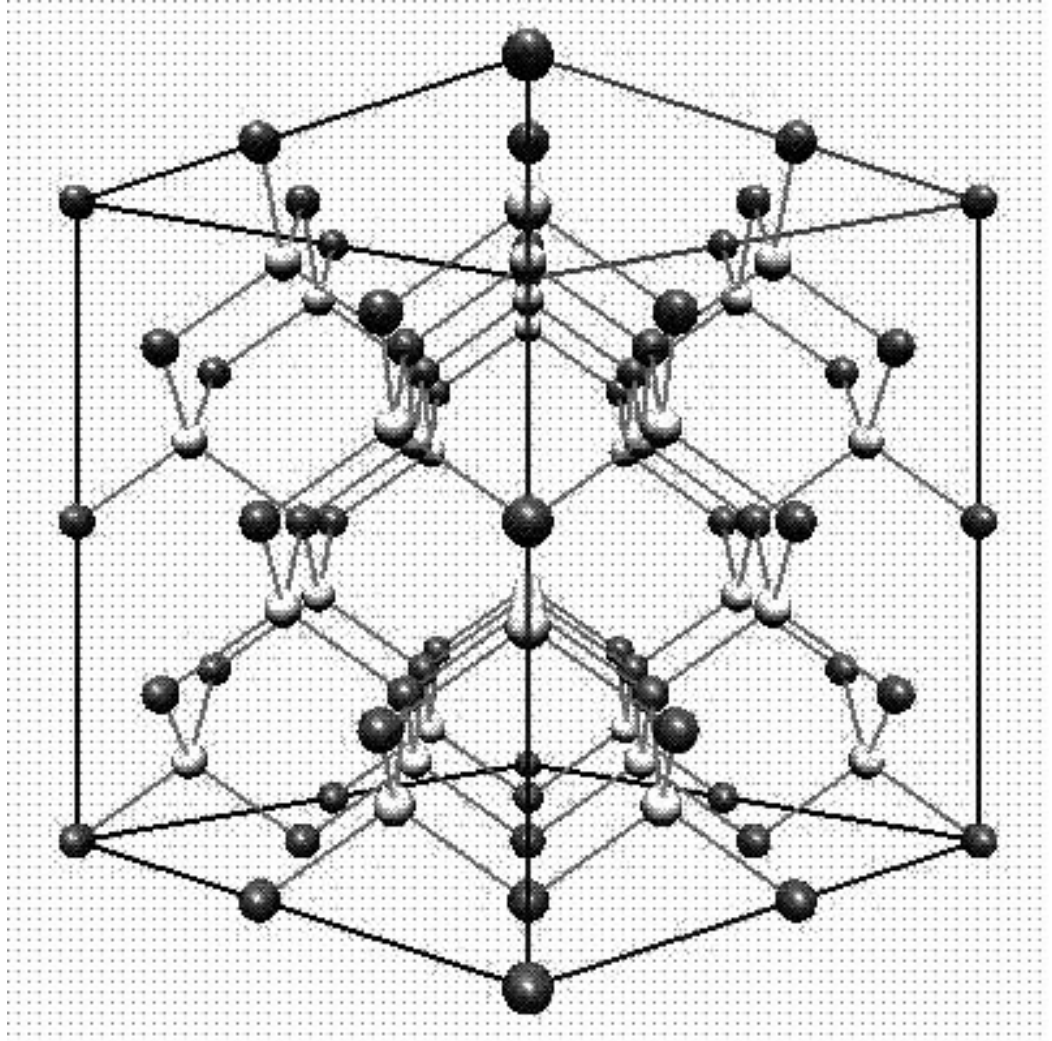

Figure 1-3: 4x4 representation of GaSb [Akopian, (2000)]. Note, the atomic radii are not the proper covalent radii for $\mathrm{Ga}$ and $\mathrm{Sb}$. 
The perfect crystal depicted above, however, does not represent the case for real ones. A crystal will always have a finite concentration of defects at a finite temperature. While it is true that adding defects to a perfect crystal increases its internal energy, the total free energy $(G)$ of a crystal at a temperature $T$ is made up of the sum of the internal energy $(H)$ and the entropy $(S)$, which becomes more negative by the addition of defects.

$$
G=H-T S
$$

Equation 1-1

The change in $G$ with the addition of $N_{x}$ defects is the difference between the increase in internal energy of the given defect $\mathrm{X}$ and the increase in the entropic term.

$$
\Delta G=\Delta H N_{X}-T \Delta S N_{X}
$$

Equation 1-2

The change in entropy is given as the entropy of mixing.

$$
\Delta S_{m i x}=-R\left(N_{X} \ln N_{X}+\left(1-N_{X}\right) \ln \left(1-N_{X}\right)\right)
$$

Equation 1-3

As the temperature of a material is reduced, a more perfect crystal is favored, but an absolutely perfect crystal is never obtained [Porter and Easterling, (2000)].

Six types of native point defects are possible in a compound semiconductor. A native point defect consists of only the species forming the matrix, in this case either $\mathrm{Ga}$ or $\mathrm{Sb}$, localized to a single atomic position, breaking the local symmetry. The first type of native point defect is a vacancy. A vacancy (V) is simply the absence of an atom on its lattice site, therefore there are two variants of vacancies, $\mathrm{V}_{\mathrm{Ga}}$ and $\mathrm{V}_{\mathrm{Sb}}$. The second type of defect is the self-interstitial (I), where a matrix atom sits in an interstitial space. $I_{\mathrm{Ga}}$ and $\mathrm{I}_{\mathrm{Sb}}$ form the two variants of this defect in this semiconductor. These spaces can be seen quite well in Figure 1-3 as the large open areas. The third type of defect, present only in compound crystals, is the anti-site defect. In this case, an atom of one species sits on a site normally occupied by the other species. Once again, there are two variants, a 
Ga-antisite $\left(\mathrm{Ga}_{\mathrm{Sb}}\right)$ and a Sb-antisite $\left(\mathrm{Sb}_{\mathrm{Ga}}\right)$, where the first atom in the notation represents the atom and the subscript represents the atomic position in a regular crystal.

The equilibrium concentrations of the native point defects can be developed from a thermodynamic treatment. The creation of a defect requires a formation energy, but as long as that is lower than the product of the temperature and the change in configurational entropy (always positive), it is energetically favorable. By minimizing the Gibbs free energy in Equation 1-2, the equilibrium concentration of a defect is found to have an Arrhenius relation of the following form (using the concentration of vacancies $C_{V}$ as an example):

$$
C_{V}=A \exp \left(-\frac{H_{V}}{k T}\right)
$$

In this equation, $H_{V}$ is the formation energy of the vacancy, $T$ is the absolute temperature, $k$ is the Boltzmann constant, and $A$ is an exponential pre-factor which is a function of the temperature independent configurational entropy. Similar equations exist for the concentrations of self-interstitial and anti-site defects.

The above development is made more complicated in semiconductors when charged defects are introduced. It has been well documented that defects in semiconductors are present not only in the neutral state, but also in various charged states. A given system is often dominated by a single type of defect and charge state at a given temperature and Fermi energy $\left(E_{F}\right)$, but the total concentration of a point defect variant is the sum over all its possible charge states. In the case of charged defects, one has to incorporate the position of $E_{F}$ into the formation energy. 
Si serves as a good example for this. Vacancies in $\mathrm{Si}$ are believed to have five possible charge states: 2-, -, neutral, + and $2+$. Experiments carried out at cryogenic temperature were used to determine the energy levels of the individual charge states (for this temperature) within the band gap of Si [Watkins, (1986)]. The situation is outlined in Figure 1-4. In order to adjust the formation energy of a defect depending on the charge state, one needs to consider the difference between the energy of the level and the Fermi energy. If the $\mathrm{Si}$ is doped n-type with the Fermi energy near the conduction band $\left(E_{C}\right)$, more negatively charged vacancies will be energetically favorable. As the $E_{F}$ moves closer to the conduction band, a larger energy gap exists between it and the positively charged vacancies. Electrons, which are in abundance under n-type doping, drop from $\mathrm{E}_{\mathrm{F}}$ to the defect level, reducing their energy. This leads to a transition towards more negatively charged vacancies. In the case of p-type $\mathrm{Si}$, the situation is reversed. This shows that the Fermi drastically affects the concentrations of the charged defects.

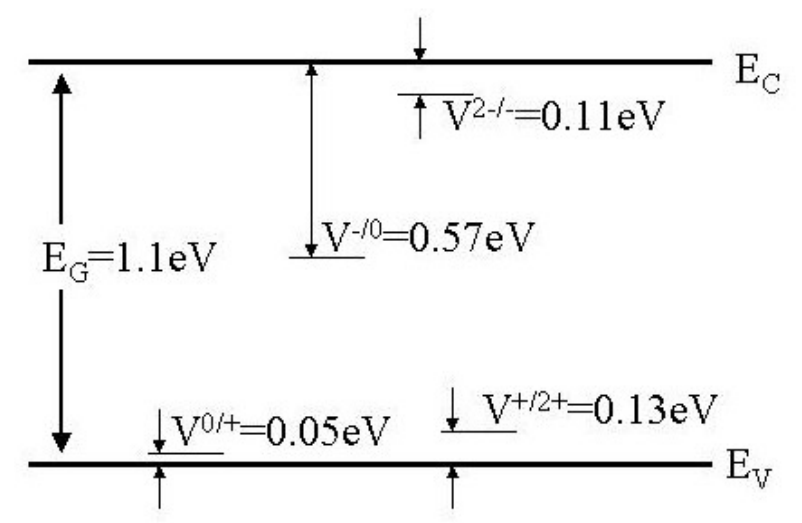

Figure 1-4: Energy levels of vacancies within the band gap of $\mathrm{Si}$

\subsection{Motivation for this Research}

The understanding of both self- and foreign atom diffusion in semiconductor systems is of fundamental importance. Diffusion of nearly all common dopants and all 
self-atoms in semiconductors is mediated by native point defects in the crystalline lattice. Diffusion remains one of only a few methods for obtaining information on point defect properties such as equilibrium concentrations, formation and migration energies, as well as charge states.

Diffusion studies in semiconductors also have a profound impact on technology. Diffusion processes govern the dopant profiles that form the junctions in all microelectronic devices. As devices are engineered to ever-shrinking dimensions and higher dopant concentrations, the understanding of diffusion becomes more and more critical to be able to maintain the abrupt, shallow junctions as required by the semiconductor road map.

The diffusion studies in GaSb reported here were originally proposed due to the comparable abundances of the two stable isotopes of the component elements [Haller, (1995)]. Diffusion studies on compound semiconductors had been carried out in the past using isotopically enriched layers (see Section 2.4.2) on GaAs, AlGaAs and GaP to name a few, yet in all these systems, diffusion could only be followed on one sublattice, namely that of Ga. This is because As and P both only have a single stable isotope. Both Ga and $\mathrm{Sb}$ have two stable isotopes, allowing for diffusion on two sublattices to be measured simultaneously for the first time.

Research in the GaSb system itself has recently intensified due to applications in high speed electronics as well as infrared lasers, detectors, and photovoltaics. Particular success has been seen in GaAsSb based DHBTs, where switching speeds of up to 300 GHz have been reported [Dvorak, (2001)]. GaSb thermo-photovoltaic (TPV) devices are currently being sold as a means of generating power from a home furnace with 
efficiencies approaching $11 \%$ by JX Crystals, Inc. When used in conjunction with InGaP and GaAs, a mechanically stacked solar cell with GaSb was recently shown to have an efficiency of 34\% [Fraas, (2001)].

\subsection{Previous Diffusion Studies}

\subsubsection{Self-Diffusion}

Eisen and Birchenall were the first to study self-diffusion in GaSb [Eisen, (1957)], using the radiotracer technique discussed in Section 2.4.1. They sputter-deposited radioactive isotopes of $\mathrm{Ga}$ and $\mathrm{Sb}$ onto separate surfaces of single crystalline $\mathrm{GaSb}$. Following annealing, the samples were measured for activity at $\sim 1 \mu \mathrm{m}$ depth steps by counting layer radioactivity and then grinding with emery paper. They did not control the partial pressures of the materials, and admittedly had difficulty in measuring the radioactivity and the grinding depths accurately, but did come up with initial values for the diffusion pre-factors as well as the activation energies.

Weiler and Mehrer published the most comprehensive study of self-diffusion in GaSb [Weiler, (1984)]. They used the technique described above, but advanced it a few steps by annealing in Ga- and Sb- rich atmospheres and using sputter sectioning. Their observations were:

- Ga and Sb diffuse relatively slowly compared to the elements of other IIIV compounds.

- The diffusivities of $\mathrm{Ga}$ and $\mathrm{Sb}$ in $\mathrm{GaSb}$ are of equal magnitude with $\mathrm{Ga}$ diffusing slightly faster.

- Both species diffuse faster in an Sb-rich environment. 
Given that $\mathrm{Sb}$ and $\mathrm{Ga}$ were found to diffuse at nearly identical rates, a coupled diffusion mechanism of the two species was proposed. Weiler and Mehrer hypothesized a triple defect mechanism, noteably $\left(\mathrm{V}_{\mathrm{Ga}} \mathrm{Ga}_{\mathrm{Sb}} \mathrm{V}_{\mathrm{Ga}}\right)^{-}$, to be the diffusion vehicle (see Figure 1-5). This hypothesis was born out of the crystal growth data which suggested the $\mathrm{V}_{\mathrm{Ga}} \mathrm{Ga}_{\mathrm{Sb}}$ double defect as being responsible for the intrinsic p-type nature of $\mathrm{GaSb}$ (as discussed in Section 1.1).

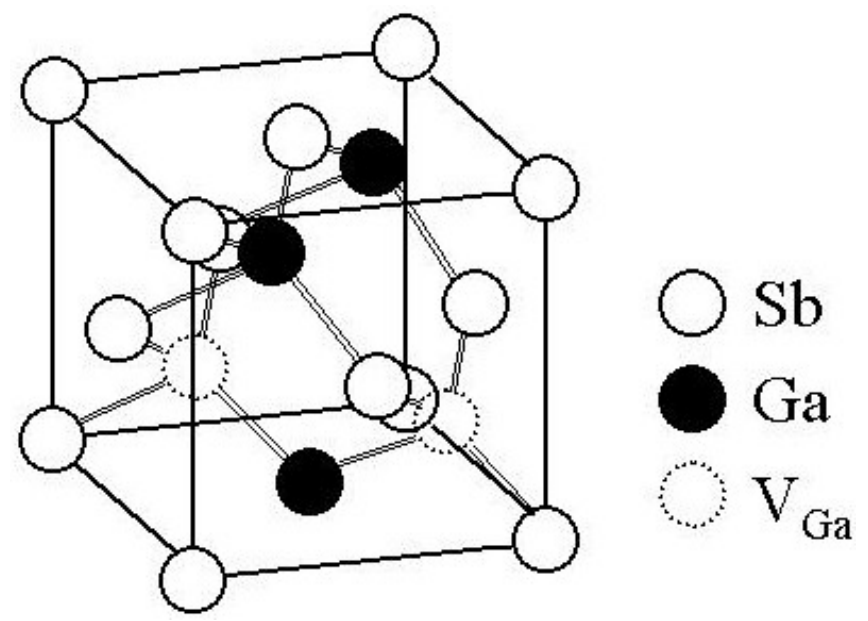

Figure 1-5: Triple defect proposed by Weiler and Mehrer for the vehicle of Ga and Sb diffusion [Mehrer, (1984)]. The defect is clearly visible towards the bottom portion of the figure with its $\mathrm{V}$ shaped configuration around the Ga antisite.

The diffusivity results from Weiler and Mehrer are shown in Figure 1-6. This plot includes the data from Eisen and Birchenall as well as data from work done by Boltaks and Gutorov on Sb self-diffusion in GaSb in 1960 [Boltaks, (1960)]. As is evidenced by this figure, self-diffusion data in GaSb vary over a very wide range. This also underlines the inherent difficulties in measuring precisely self-diffusion data, and in these cases, particularly through the radiotracer technique. 


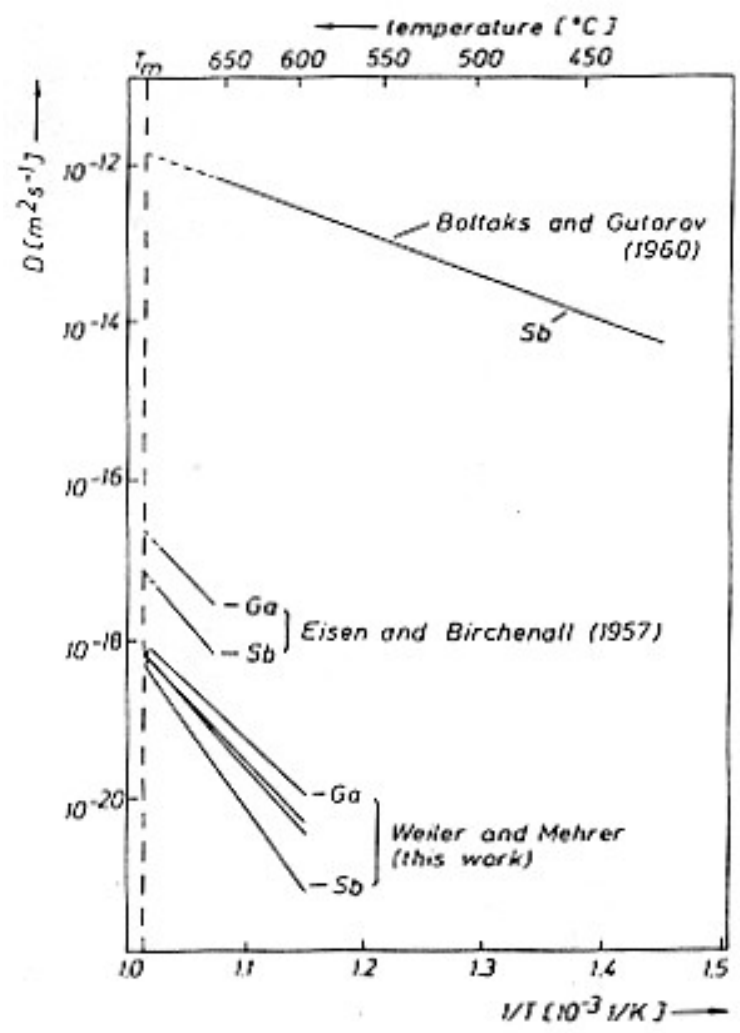

Figure 1-6: Self-diffusivites of Ga and $\mathrm{Sb}$ in GaSb as published by Weiler and Mehrer (1984).

\subsection{2 $\underline{\text { Zn Diffusion }}$}

Zn diffusion studies in GaSb date back to Kyuregyan and Stuchebnikov, who were the first to publish any data on the subject [Kyuregyan, 1971]. They showed that $\mathrm{Zn}$ diffusion from a constant surface concentration source cannot be described by the complementary error function fit. Instead, the diffusion coefficient is a function of the zinc concentration in the sample. Specifically, they found a quadratic dependence of $D$ on the zinc concentration. They were quick to draw similarities to $\mathrm{Zn}$ diffusion in GaAs, where $\mathrm{Zn}$ diffusivity has a similar dependence. Since this first publication, many groups have published results further exploring these initial findings, yet no clear identification of the diffusion mechanism has been set forth [Da Cunha, (1974); Sundaram, (1993); Conibeer, (1994); Conibeer, (1996); Bett, (1997); Mimkes, (1998)]. Conibeer et al. 
proposed in 1996 that Zn diffuses via a substitutional-intersititial mechanism, yet lacked sufficient evidence to support either the dissociative (Frank-Turnbull [Frank, (1956)]) or kick-out (Gösele-Morehead [Gösele, (1981)]) mechanism.

Two characteristic Zn diffusion profiles in GaSb have been observed by previous research groups. The most widely observed profile is the "kink-and-tail" profile [Sundaram, (1993); Conibeer, (1994); Conibeer, (1996); Bett, (1997)], identifiable through its double hump shape. A second type of $\mathrm{Zn}$ diffusion profile observed in $\mathrm{GaSb}$ is the "box" profile [Kyuregyan, (1971); Dakshinamurthy, (1999)], identifiable by its flat diffusion profile followed by a steep drop at the diffusion front. Qualitative representations of these two types of profiles are shown below. Only one group has published results showing both types of profiles [Da Cunha, (1974)].

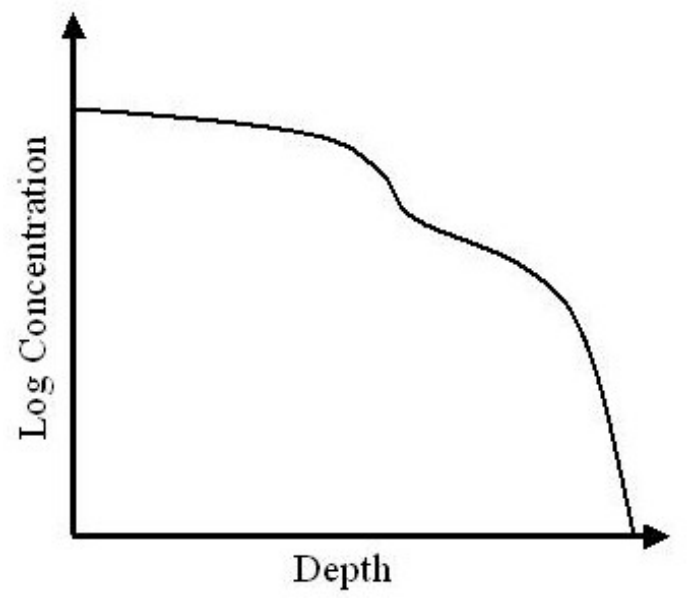

Figure 1-7: Qualitative example of the kink and tail profile. The kink section is clearly visible behind the diffusion front.

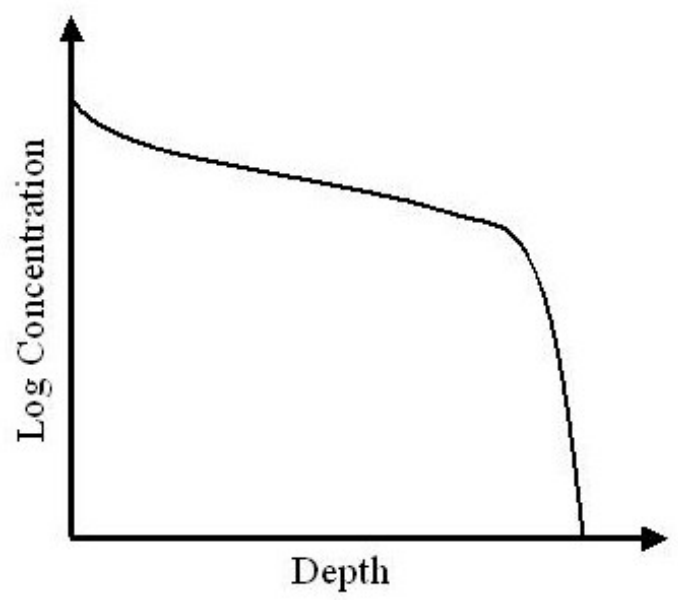

Figure 1-8: Qualitative example of the box profile. No kink is present in this profile showing a steep diffusion front.

Two hypotheses have been put forth to explain the kink-and-tail profile. The first explanation, given by Da Cunha and Bougnot, is a Fermi level driven effect drawn from 
studies on $\mathrm{Zn}$ diffusion in GaAs. They claim that as the Fermi level is driven lower and lower by the in-diffusion of $\mathrm{Zn}$ acceptors, the formation of compensating donors in the form of $\mathrm{Zn}$ interstitials becomes energetically favorable. The ionized donors diffuse as an electrically active species much more slowly than the non-ionized states of $\mathrm{Zn}$ in the lattice. A second hypothesis for the kink-and-tail profile comes from Conibeer et al. [Conibeer, (1994)]. They propose that $\mathrm{Zn}$ diffuses in the form of electrically inactive dimers and trimers at high concentrations, leading to a Fickian diffusion profile until their concentration drops to below that of $\mathrm{Zn}_{\mathrm{Ga}}$.

A single study by TEM of extended defects caused by $\mathrm{Zn}$ diffusion into GaSb at very high surface concentrations and temperatures was carried out in 2000 [Jäger, (2000)]. It found a diffusion region full of large dislocation loops, as well a network of dislocations and precipitates. Through analysis of the inside-outside diffraction contrasts in bright-field TEM images and EDX spectroscopy, the dislocation loops were all found to be of the Ga self-interstitial type. This is consistent with $\mathrm{Zn}$ diffusing via the kick-out mechanism at these high concentrations. 


\section{Diffusion}

Diffusion refers to the spontaneous intermingling of particles as a result of random thermal motion. In crystalline semiconductors diffusion can be broken down into three distinct categories. The most basic is self-diffusion. This is the random movement of constituent atoms in a lattice populated solely by that species. The second level of diffusion is interdiffusion of two distinct species across an interface. The third category of diffusion is impurity diffusion where foreign chemical species move in very low concentrations through a host lattice. A great deal of work has been done on diffusion, developing a mathematical framework to describe it, experimental methods to measure it, and modeling work to extract the diffusion parameters of diffusivity, activation and migration energies, entropy of diffusion, as well as equilibrium point defect concentrations.

\subsection{Development of Diffusion Theory}

In 1855, Adolf Fick, Professor of anatomy at the University of Zürich, formulated Fick's Laws of diffusion, in essence converting Fourier's Law of heat transfer to concentration gradients instead of heat gradients [Fick, (1855)]. Fick used this model to formally describe the movement of atoms in liquid solutions, yet it is applicable to all types of diffusion studies and forms the foundation for all mathematical analyses. Fick's First Law states that the diffusion flux $J$ is proportional to the gradient in the concentration. 


$$
\vec{J}=-D \vec{\nabla} C
$$

Equation 2-1

Fick's Second Law states that the change in the concentration as a function of time is equal to the gradient in the diffusion flux.

$$
\frac{\partial C}{\partial t}=-\vec{\nabla} \vec{J}=\vec{\nabla}(D \vec{\nabla} C)
$$

Most of the current and interesting work on diffusion in semiconductors focuses on the quantity $D$. Henry Eyring [Glasstone, Eyring (1941)] was the first to apply transition state theory to diffusion in solids, leading to today's common Arrhenius plots, depicting the exponential dependence of $D$ on temperature, as depicted in the first part of the equation below. An energy barrier of height $Q$ must be overcome in order for diffusion to occur. The pre-exponential factor $D_{0}$ in Equation 2-3 was expanded by applying the elastic model of atomic interactions. The lattice parameter $a$, a constant $g$ specific to the crystal type, and the attempt jump frequency of an atom about its equilibrium position in the jump direction $v_{0}$, were all added to form the common form seen today.

$$
D=D_{0} \exp \left(-\frac{Q}{k T}\right)=g a^{2} v_{0} \exp \left(\frac{S}{k}\right) \exp \left(-\frac{H}{k T}\right)
$$

In the above relation, $S$ is the entropy and $H$ is the enthalpy.

For diffusion to occur in a crystal there must be either vacancies (open spaces for atoms to move into) or mobile interstitials (atoms located in interatomic spaces). As will be discussed in Section 2.3, diffusion in extended-defect-free crystals is mediated by these two types of defects through various distinct mechanisms. In Section 1.2 it was stated that point defects are present in crystals at all temperatures, implying that finite diffusion exists at all temperatures. 
Longini and Greene were the first to incorporate the effect of charge states into diffusion theory [Longini, (1956)]. As discussed in Section 1.2, charged defects can be favored or suppressed depending on the Fermi level position in a semiconductor. They established a simple equilibrium reaction between an uncharged and a charged defect, for example in the case of a vacancy $V$ :

$$
V^{0}+j e^{-} \Leftrightarrow V^{j-}
$$

Equation 2-4

An uncharged vacancy picks up $j$ electrons, making it negatively charged by the same factor. General chemistry requires that at equilibrium, there exists a reaction constant that is a function of the concentrations of the species. In this case:

$$
K=\frac{\left[V^{j-}\right]}{\left[V^{0}\right]\left[e^{-}\right]^{j}}
$$

The electron concentration $\left[e^{-}\right]$in intrinsic semiconductors is given by

$$
n_{i}{ }^{2}=N_{C} N_{V} \exp \left(-\frac{E_{G}}{k T}\right),
$$

where $N_{C}$ and $N_{V}$ are the density of states of the conduction and valence bands respectively. In extrinsic semiconductors, the electron concentration becomes $n$, given by

$$
n=N_{C} \exp \left(-\frac{E_{C}-E_{F}}{k T}\right) .
$$

Dividing the rate constants for the intrinsic and extrinsic cases, the following relation results:

$$
\left[\begin{array}{c}
V^{j-} \\
{\left[V^{0}\right]}
\end{array}=\exp \left(-\frac{E_{V^{0 /-}}-E_{F}}{k T}\right)\right.
$$


This theory is used to model the impact of the Fermi level on the concentration of charged point defects.

Much of the development in diffusion has come from studies in metals where vacancies mainly dominate self-diffusion. In contrast to metals, in semiconductors one cannot neglect the contribution of self-interstitials to self-diffusion. This can be attributed to the difference in crystalline structure of the two materials. Metals crystallize in a close packed lattice where very little open space exists between atoms. Rather than being a very tight and closely packed system, the lattice in semiconductors is relatively open.

\subsection{Driving Forces of Diffusion}

Diffusion is a thermodynamically driven event. The two factors that dictate how diffusion progresses are minimization of free energy and random walk.

Minimization of free energy is reached when the chemical potentials of all the species within a material are equal. In most diffusion problems considered in semiconductors, the chemical potential $\mu$ is directly proportional to the concentration $C$ of a given species, an assumption used in the development of Fick's Laws in the previous section. In these cases, self-atoms and impurity atoms are perfectly miscible (up to the solubility level), allowing Fick's Laws to be applied with simple concentration variables. The driving force for diffusion is thus stated as the gradient in concentration, though this is a simplification of the true driving force, namely the gradient in chemical potential. If however the diffusing species prefer like neighbors, then $\partial \mu / \partial C$ can be a negative value at low temperatures, leading to the possibility of "uphill" diffusion. This type of diffusion has not been observed in either self- or $\mathrm{Zn}$ diffusion in $\mathrm{GaSb}$. 
When the material is in equilibrium (free energy mimimum), there still exists some diffusion through random walk. Although the chemical composition of the material does not change, the atoms still have thermal energy which causes them to vibrate and occasionally move from their atomic position. The random walk process is therefore not a chemically driven effect, but one driven only by thermal energy.

The most basic form of diffusion is self-diffusion. Self-diffusion is the movement of the species atoms making up the compound of interest. In self-diffusion, no chemical gradient exists, so the process is governed purely by random walk of the individual atoms. Without a means to mark an individual atom separately from the bulk, selfdiffusion is a process which cannot be directly observed. While a volume may remain constant with no change observable from the outside, extensive diffusion could be taking place. As will be discussed in Section 2.4, the problem of labeling and measuring selfdiffusion has been overcome through nuclear marking of certain atoms, allowing for marking without changing the chemical nature of the atom.

Interdiffusion is the second type of diffusion in semiconductors. In this case, a junction clearly separates two regions. Random walk and chemical effects dictate whether interdiffusion of the two regions occurs. In most cases, the free energy of the crystal will be reduced by intermixing of the two regions, leading to interdiffusion governed by Fick's Laws with concentration gradients as the driving force.

The final type of diffusion important for semiconductors is impurity diffusion, and more specifically, dopant diffusion. Dopant species are usually introduced to the semiconductor either during growth (for homogenous doping) or during processing steps, such as implantation. The large chemical gradient required for the junction is a driving 
force for homogenization of the dopant species. This is usually something that is avoided, particularly when an abrupt junction between $p$ and $n$ regions is desired. What makes dopant diffusion particularly complicated and interesting is the dependence of the charge state on the Fermi energy. Dopants are usually added which act at room temperature either as donors (n-type) or acceptors (p-type). Nearly any common dopant will be ionized at a temperature where diffusion becomes appreciable, altering the Fermi level, and thus the diffusion behavior.

\subsection{Microscopic Mechanisms of Diffusion}

The point defects discussed in Section 1.2 are the key to understanding diffusion in semiconductor crystals. While diffusion can and does occur through extended defects, such as grain boundaries and dislocations, these defects are suppressed in semiconductors devices to maximize performance. Diffusion can thus be thought of as the statistical movement of individual atoms within a periodic lattice structure.

A number of mechanisms have been proposed to explain both self- and foreign atom diffusion behavior in crystals, and these can be grouped into two categories: direct and indirect. As shall be explained, direct diffusion mechanisms do not involve any native point defects, while indirect mechanisms require point defects for atomic movement.

The most simple type of mechanism is a simple exchange, or ring mechanism. This direct mechanism in the case of self-diffusion is sketched in Figure 2-1. As can be readily seen, the mechanism involves the simultaneous swapping of atomic positions, or a coordinated movement of many atoms at once. Substitution a self-atom with a foreign species would give rise to an example of this kind of mechanism for foreign atom 
diffusion. No experimental evidence has been confirmed which supports this mechanism for either self- or foreign atom diffusion.

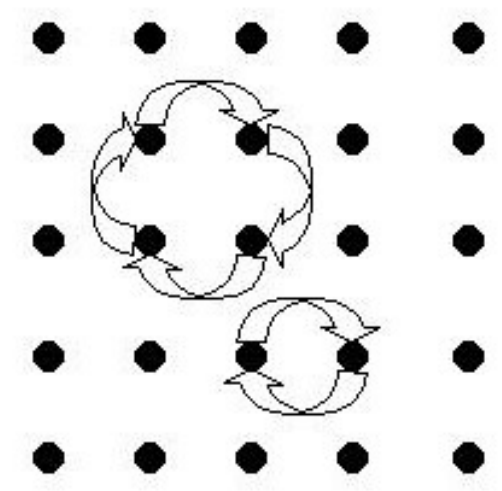

Figure 2-1: Ring and exchange mechanisms for self-diffusion.

A second type of direct diffusion mechanism is the pure interstitial mechanism, diagramed in Figure 2-2. This type of diffusion has been observed only in foreign atom diffusion where the foreign species is completely dissolved interstitially, such as hydrogen or lithium in silicon or germanium. The problem with impurities diffusing according to this mechanism is that they are highly mobile at even modest temperatures and cannot be used to form the junctions required by typical device structures.

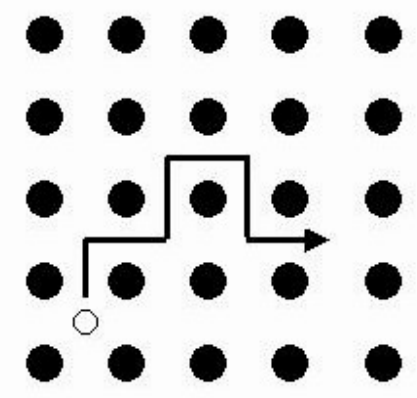

Figure 2-2: Pure interstitial mechanism for a small foreign atom $(0)$ diffusing interstitially through a host lattice matrix. 
Of more practical importance for semiconductors are the indirect diffusion mechanisms. While understanding pure interstitial diffusion is important for limited applications, self- and dopant atom diffusion is of much greater interest. The large majority of these species diffuse via indirect diffusion mechanisms and require native point defects in the semiconductor for movement.

A first example of an indirect mechanism is the vacancy mechanism. As its name implies, vacancies are the mediating native point defect. In this mechanism, diffusion proceeds through movement of vacancies. As shown below, a vacancy approaches an impurity atom occupying a substitutional site. The atom on the substitutional site can exchange its position with the vacancy, thereby moving by one lattice location. The vacancy is attracted to the defect due to total energy reduction through strain relief or coulomb attraction. In order for the foreign atom to continue to diffuse, the vacancy must dissociate itself from the impurity and approach it again from a different side, or another vacancy must take part. This mechanism has been found to be applicable for selfdiffusion as well as large impurity diffusion in metals where there is relatively little interatomic space for interstitials and where there is an abundance of vacancies.

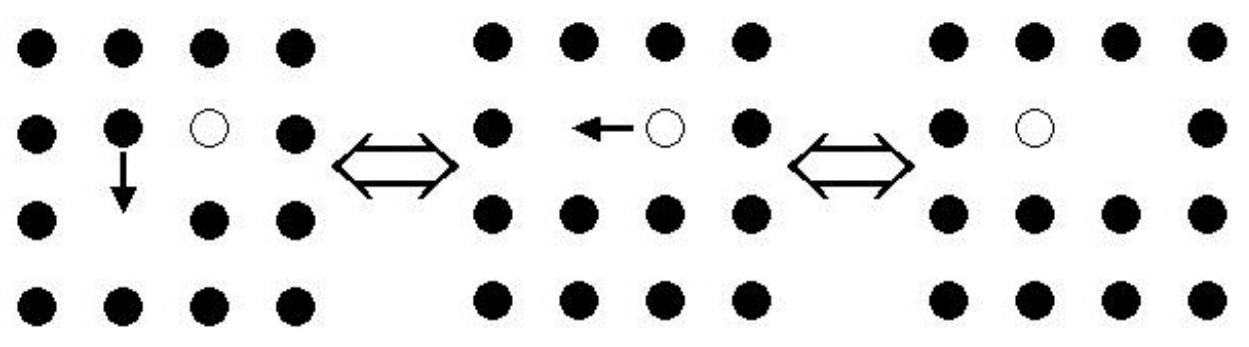

Figure 2-3: Vacancy mechanism mediating the diffusion of a large impurity atom $(\circ)$ in a host matrix. 
The interstitialcy mechanism in Figure 2-4 is analogous to the vacancy mechanism, except in this case an interstitial is the point defect which mediates diffusion. Each movement of the interstitial allows an atom to move by a single atomic position. Long range diffusion can be achieved through either many interstitials moving the atom one atomic distance each, or a single self-interstitial which can move the atom through a circular motion around it. This mechanism has been used to explain the diffusion of both self- and foreign atoms. Similar to the vacancy mechanism, self-interstitial-impurity pairs are often energetically favorable due to strain and charge considerations.

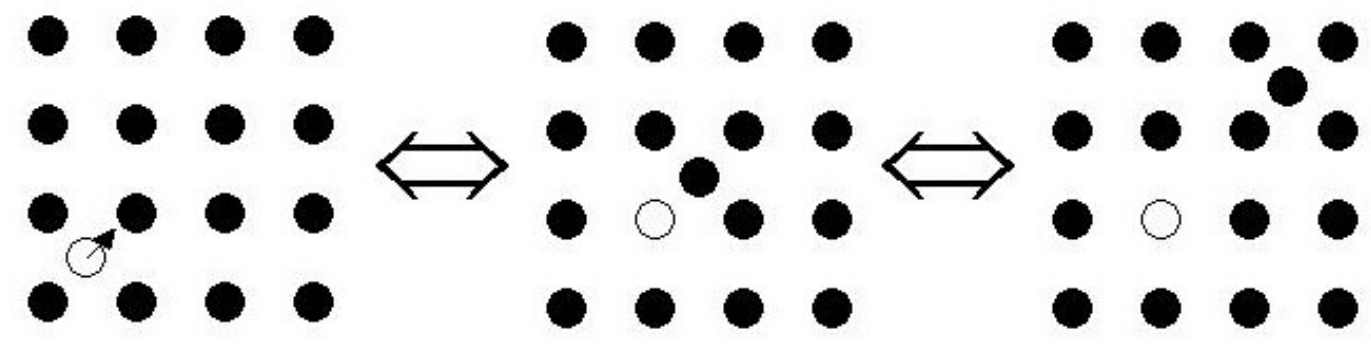

Figure 2-4: Interstitialcy mechanism mediating the diffusion of an impurity atom $(\circ)$ in a host matrix.

Additional diffusion mechanisms for dopant elements in semiconductors are the substitutional-interstitial mechanisms. The kick-out mechanism is illustrated in Figure 2-5, while the dissociative (Frank-Turnbull) mechanism can be seen in Figure 2-6. The difference between the kick-out and the interstitialcy mechanism described above is that the interstitial foreign atom can migrate rapidly over long distances (more than a single atomic position) before it kicks out a matrix atom into the interstitial position, forming a new interstitial. In a similar manner, the dissociative mechanism involves an interstitial foreign atom that can migrate over long distances before finding a free vacancy with which it can react to return to a substitutional position. 


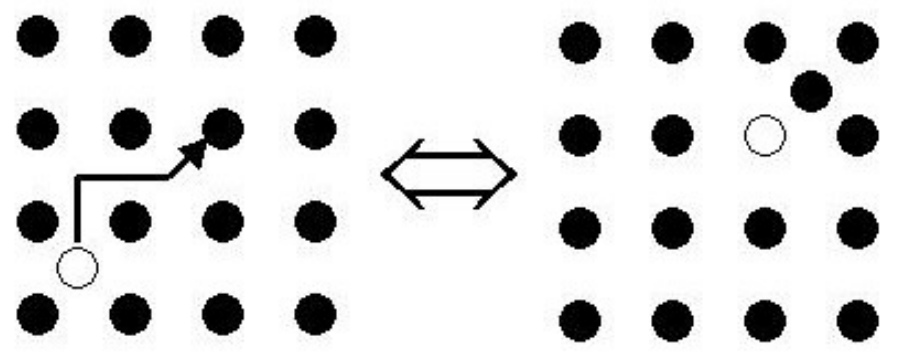

Figure 2-5: Kick-out mechanism mediating the diffusion of an impurity atom $(\circ)$ in a host matrix.

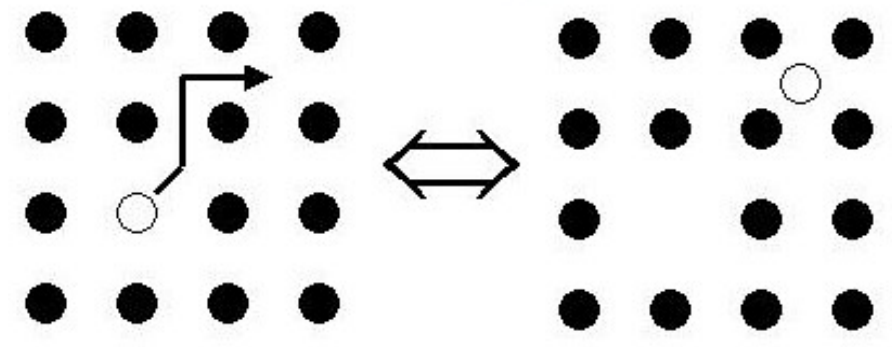

Figure 2-6: Dissociative mechanism mediating the diffusion of an impurity atom $(\circ)$ in a host matrix.

While the diagrams of the diffusion mechanisms are a useful way to think of the similarities and differences of each mechanism, the individual reactions are the basis of the mathematical expressions used in the modeling of the diffusion processes. The mechanisms for foreign atom diffusion mediated by the four indirect mechanisms are given in the following reactions in the following order: vacancy, interstitialcy, kick-out and dissociative mechanisms.

$$
\begin{aligned}
& A_{s}+V \Leftrightarrow A V \\
& A_{s}+I \Leftrightarrow A I \\
& A_{s}+I \Leftrightarrow A_{i} \\
& A_{s} \Leftrightarrow A_{i}+V
\end{aligned}
$$

Equation 2-12 
Self-diffusion is mediated by Equations $2-9$ and $2-10$. In this case, $A$ represents a tagged self-atom. In these equations, the defect-substitutional atom pair must dissociate in order for diffusion to continue.

Which mechanism is dominant in a given material system at a particular temperature, either for self- or foreign atom diffusion, is very dependent on the native defects which are present in the crystal at the diffusion temperature. As can be clearly seen by the diagrams, the concentration of vacancies or self-interstitials will have a large influence on diffusion rates. For example, one can easily imagine that the dissociative mechanism would not be very efficient if the formation energy of vacancies were high.

\subsection{Experimental Methods for Measuring Diffusion}

Diffusion studies in solids require careful control of numerous parameters as well as the quantification of the diffusing species. Temperature and pressure control are well understood and the techniques for controlling them are well established and do not require further discussion. However, the means of identifying and quantifying the diffusing species poses a difficult problem, and numerous methods have been developed which do require discussion.

In both the radiotracer and stable isotopic methods, the diffusing species can be identified as separate from the matrix, even in self-diffusion studies. In the first case, the radioactivity of the atom serves as a marker, while in the second it is the atomic mass. Both methods offer a clear marking scheme, while maintaining identical chemistry with the matrix. 


\subsection{1 $\quad$ Radiotracer Methods}

Kheveshi and Zagrubski introduced the use of radioactive isotopes in solid-state diffusion studies in the early 1920's [Bokstein, (1985)]. The technique, however, did not become widely implemented until after World War II, when nuclear reactors began to serve as production facilities for radioactive isotopes. Since then a great deal of diffusion research has been carried out using this technique, with particular success in the area of self-diffusion.

From the 40's and into the 70's, a significant amount of research was invested in documenting all the isotopes for each element, including half-lives of the radioactive species. Diffusion studies were just one branch of science that benefited tremendously from this newfound availability of radioisotopes. The technique is carried out by introducing a certain concentration of the species of interest on or near the surface of the diffusion matrix material. The radioactivity of the element serves as a means of quantification, while the electronic and chemical configuration of the atom is identical to that of the stable isotopes of the same element. Following a thermal annealing step, the radioactivity of the sample as a function of depth is measured. From this, one can extract a concentration profile, correcting for the decay rate of the isotope.

Radiotracer studies proved to be a powerful way to extract diffusion data from numerous systems, but as with all methods, it has its limitations. The half-lives of the available radioactive isotopes limit which systems can be studied. In cases of a short half-life (e.g. ${ }^{31} \mathrm{Si}$ ), the experiments are forced to be completed very quickly, limiting them to high temperature, short time anneals. They must also be carried out very close to where the isotope is created so that it is not all has decayed before the experiment begins. 
Too low of a specific activity poses a different problem. Low activity provides few counts which can be used to extract the concentration of the diffusion species. Another drawback is that all the diffusion experiments have to be done from the surface into the bulk since the radiotracers must be deposited prior to annealing. Surface deposition is always susceptible to creating defects (e.g. implantation damage) or contamination (e.g. oxidation, nitridation, metals) which can drastically affect the diffusion rate. Lastly, safety has become a major concern with radioactive materials. Processing, handling, and disposing of them in a safe manner, let alone the bureaucracy that is involved in obtaining permits to have them, often limit the applications.

Regardless of the problems discussed, radiotracer studies form the vast bulk of self-diffusion data currently available for solids.

\subsubsection{Isotopically Enriched Samples}

An alternative to the radiotracer method is using stable isotopes as a means of identifying a diffusing element in self-diffusion studies. Nearly $80 \%$ of the elements on the periodic table have multiple stable isotopes found in nature. The isotopic composition of the elements is relatively constant around the world. One example is carbon. Carbon in its natural form is $99 \%{ }^{12} \mathrm{C}$ and $1 \%{ }^{13} \mathrm{C}\left({ }^{14} \mathrm{C}\right.$ is a radioactive isotope that is used to date organic materials). To use stable isotopes as diffusion markers, one must differentiate the diffusing species from the bulk material, so an isotopically enriched or depleted source must be procured.

Nuclear weapons research provided the technology to make this method of research feasible. Fusion bombs require specific isotopes of uranium or plutonium to work, so the nuclear powers poured tremendous amounts of money into building gas 
centrifuges to enrich uranium mined at its natural composition, or plutonium from spent uranium in nuclear reactors. The gas centrifuges are able to enrich any kind of isotope that can form a gaseous chemical species, but the cost that the respective governments charged until just recently for macroscopic amounts of enriched isotopes made experiments unfeasible.

This all changed with the fall of the Soviet Union. With the weapons industry seriously cut back, the Russian government found itself with a tremendous enriching ability, but no outlet for products. The scientists who had once made materials for bombs began enriching other materials that could be used for scientific studies, including diffusion work. Prices for isotopically pure substances fell drastically, and a wide range of solid state studies, including diffusion, became viable [Haller, (1995)].

In this technique, the atomic mass is used as the label by creating regions of isotopically pure material and following their diffusion into either natural material or another layer of material enriched with a different isotope. Ideal sample structures have isotope junctions between layers showing greater than three orders of magnitude difference between isotopic concentrations, allowing for accurate modeling of the diffusion. The samples are usually grown as isotopically pure layers on a natural wafer with a capping layer of natural isotopic abundance. This has the benefit of moving the diffusion area of interest away from the surface and into the bulk itself. Analysis of the samples is achieved using Secondary Ion Mass Spectroscopy (SIMS) which can very effectively differentiate between single atomic masses (see Section 7.1). This technique has already been successfully applied to diffusion studies in a number of systems 
including Ge [Silveira, (1997); Fuchs, (1995)], GaAs [Wang, (1996)], GaP [Wang, (1997)], Si [Bracht, (1998)], and $\mathrm{Al}_{\mathrm{x}} \mathrm{Ga}_{1-\mathrm{x}} \mathrm{As} / \mathrm{GaAs}$ [Bracht, (1999)].

An example of this method is shown below for the case of Si. As can clearly be seen in the figure, a sample was grown with regions of enriched isotopic composition, in this case a single layer of enriched ${ }^{28} \mathrm{Si}$ (depleted of ${ }^{30} \mathrm{Si}$ ) sandwiched between layers of natural isotopic composition. The plot shows the measured concentration of ${ }^{30} \mathrm{Si}$ in the structure, both before and after a high temperature anneal. The structure allows interdiffusion across two interfaces to be measured in the form of ${ }^{30} \mathrm{Si}$ diffusion into the depleted region. A self-diffusion coefficient for $\mathrm{Si}$ was accurately extracted at this temperature [Bracht, Habilitationsschrift (2001)].

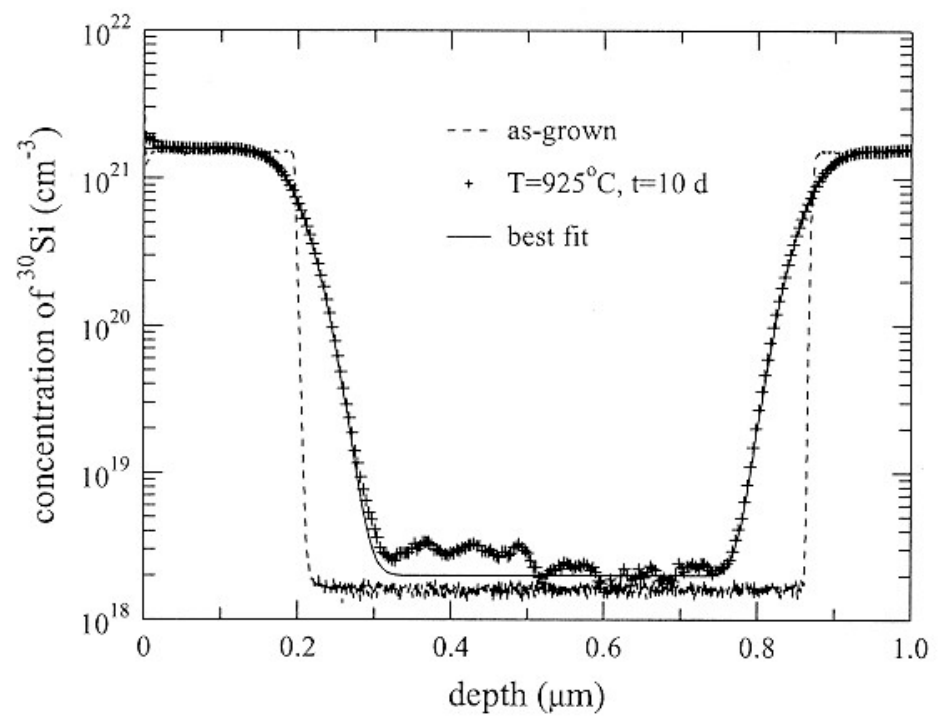

Figure 2-7: Measurement of self-diffusion in $\mathrm{Si}$ using a depleted ${ }^{30} \mathrm{Si}$ region (enriched ${ }^{28} \mathrm{Si}$ ) sandwiched between regions of natural concentration. By measuring the indiffusion of ${ }^{30} \mathrm{Si}$ from the natural regions, the self-diffusivity of $\mathrm{Si}$ at $925^{\circ} \mathrm{C}$ was found to be $5.76 \times 10^{-18} \mathrm{~cm}^{2} \mathrm{~s}^{-1}$. [Bracht, Habilitationsschrift (2001)]

As with the radiotracer method, the use of isotopically enriched substances for diffusion studies have their own drawbacks. The first is that not all elements have multiple stable isotopes. Of the elements that are interesting for semiconductor 
applications, the notable ones without multiple stable isotopes are aluminum, phosphorus and arsenic. Self-diffusion studies with these elements are limited to the radiotracer method. Furthermore, the detection limits for stable isotopes is much higher than for that of radioactive isotopes.

While macroscopic amounts of the enriched elements can be purchased for scientific use, experiments are generally limited to growing thin layers on natural material by either molecular beam epitaxy (MBE) or chemical vapor deposition (CVD). While diffusion from the surface can be avoided by growing junctions with cap layers, contamination is still an issue. Furthermore, the growth temperatures of thin film techniques are lower than the melting temperature of the materials. During growth at these low temperatures, a concentration of native point defects can be frozen in which considerably exceeds the concentration of these defects under thermal equilibrium conditions. As a consequence the self-diffusion in these structures can be significantly affected. Heat treatments to anneal out the defects are often avoided because they cause diffusion to begin in structures where abrupt junctions are necessary for accurate measurements.

\subsubsection{Closed Ampoule Annealing}

The most common way to control the atmosphere in which an annealing experiment is carried out is using the so-called closed ampoule. A schematic of a closed ampoule is shown below. Semiconductor grade quartz tubing is sealed at one end, forming the shell of the ampoule. A plug is formed in a similar fashion using tubing with a slightly smaller outer diameter than the inner diameter of the shell. 


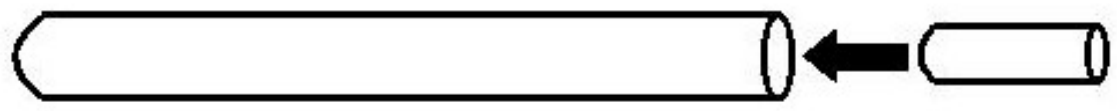

Figure 2-8: Schematic of an ampoule shell and plug.

Solid samples are placed in the ampoule shell at the sealed end. The plug is then inserted and a seal is made between the plug and shell using a hydrogen-oxygen torch.

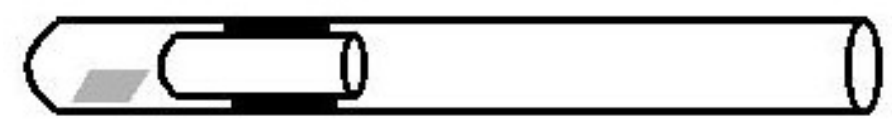

Figure 2-9: Schematic of sealed ampoule with gray semiconductor diffusion sample sealed in small volume.

Prior to sealing, any desired diffusion source can be added. The annealing ambient is controlled through a sealing station. The sealing station has the option to pump down on the ampoule and/or add ambient gases prior to the actual sealing.

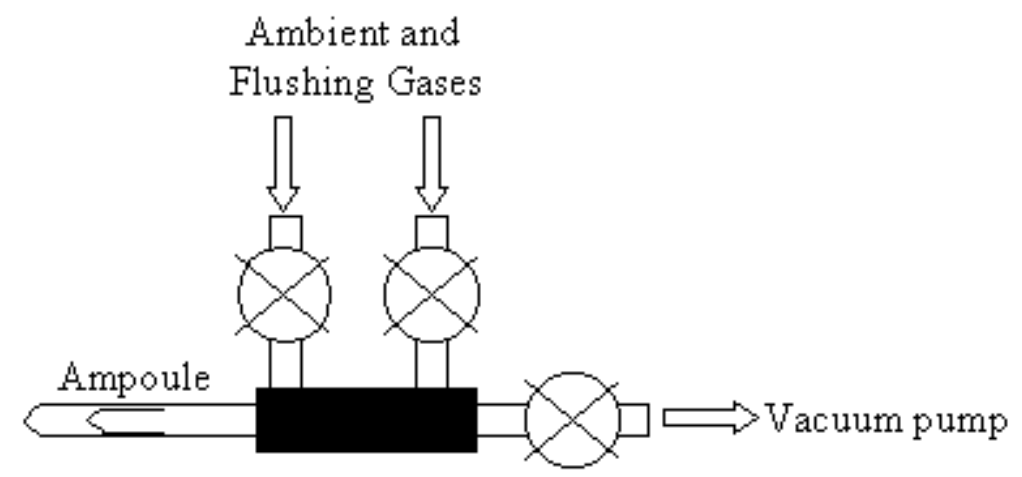

Figure 2-10: Schematic of ampoule sealing station.

Though this method is tedious for preparing samples on the industrial scale, it is a very simple way to control the temperature, pressure and atmosphere for a diffusion experiment. Care must be taken to maintain surfaces which do not impose nonequilibrium point or extended defect concentrations. Impurity diffusion using an elemental source will often lead to clustering or the creation of extended defects due to 
the injection of native point defects. Since annealing is often carried out at high temperatures, reactive gases such as oxygen are avoided. Reactions at the surface, such as oxidation, cause significant changes of the equilibrium concentration of point defects within the sample.

In self-diffusion experiments, the samples are placed in the ampoule, either under vacuum or in an atmosphere of inert gas. Extra amounts of the components making up the material can be added in order to provide an atmosphere rich in a specific species, or poor in another.

For impurity diffusion studies, one can either apply the species directly onto the surface through a deposition step (or through implantation), or diffusion can be achieved through a gas source. Many different diffusion sources have been used in the past to control the surface concentration of the diffusing species, ranging from solid to gas. A thorough understanding of the partial pressures of the various species as a function of temperature is required to quantitatively predict the surface concentrations from which impurity diffusion is driven.

\subsection{Diffusion Analysis and Modeling}

Hartmut Bracht carried out all the modeling for both the self- and $\mathrm{Zn}$ diffusion in GaSb. The development that follows is a summary of the mathematical framework he used to carry out the fitting described in the results and discussion. 


\subsubsection{Self-Diffusion}

Starting with Fick's Second Law, the solution for diffusion across an interface can readily be calculated as a slightly modified error-function solution, incorporating the concentrations of the two regions.

$$
C(x)=\frac{C_{1}+C_{2}}{2}+\frac{C_{1}-C_{2}}{2} \operatorname{erf}\left(\frac{x-d_{1}}{2 \sqrt{D t}}\right)
$$

In the equation above, $C_{1}$ and $C_{2}$ are the concentrations of the diffusion species in regions 1 and 2 , and $d_{1}$ is the distance that the interface is located from zero (usually taken to be the surface), or in other words, the depth of the interface from the surface.

In these experiments, isotopically enriched heterostructures were used to study diffusion, giving rise to not one, but three interfaces. As example of an ideal structure, the concentration profile of a single species to be studied (for example a single isotope of $\mathrm{Ga}$ ) from an as-grown wafer is shown in Figure 2-11. Growth direction in this case is from right to left, with the sample surface being at the left edge of the plot. The samples were grown on a natural composition buffer layer of concentration $C_{l}$, followed by a depleted layer $\left(C_{3}\right)$ of thickness $d_{3}$ onto which an enriched layer $\left(C_{2}\right)$ of thickness $d_{2}$ was grown. A capping layer was grown of natural isotopic composition $\left(C_{l}\right)$ on top of the heterostructure to contain the area of diffusion away from the surface. 


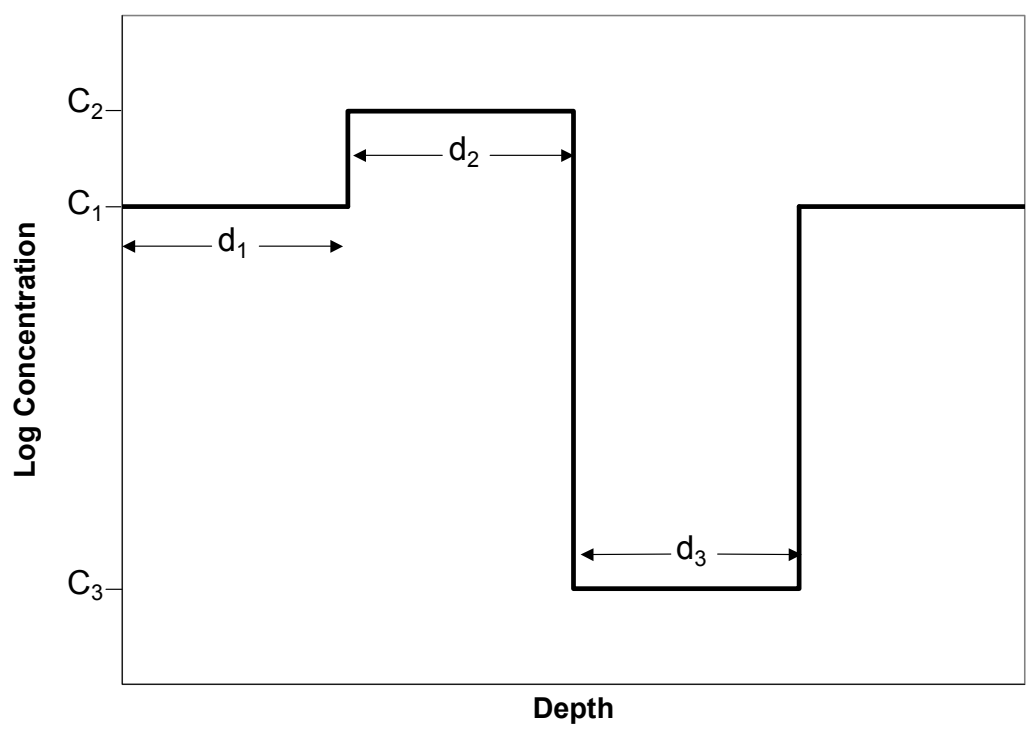

Figure 2-11: Idealized SIMS plot of as-grown isotopic heterostucture.

In this case, Equation 2-13 needs to be modified in order to account for the fact that there are three interfaces and not one. The solution is effectively a superposition of three interfaces into a single equation, shown below:

$$
C(x)=C_{1}+\frac{C_{2}-C_{1}}{2} \operatorname{erf}\left(\frac{x-d_{1}}{2 \sqrt{D t}}\right)+\frac{C_{3}-C_{2}}{2} \operatorname{erf}\left(\frac{x-d_{1}-d_{2}}{2 \sqrt{D t}}\right)+\frac{C_{1}-C_{3}}{2} \operatorname{erf}\left(\frac{x-d_{1}-d_{2}-d_{3}}{2 \sqrt{D t}}\right)
$$

Equation 2-14

In practice, diffusion profiles are fit to the above equation using $2 \sqrt{D t}$ as the fitting parameter. Once this is done, knowing the time, a diffusivity can be obtained for the specific annealing temperature.

\subsubsection{Zn Diffusion}

The development of $\mathrm{Zn}$ diffusion via the kick-out mechanism mediated by neutral Ga self-interstitial defects will now be developed. Diffusion mediated through defects of differing charge state and/or mechanism follow a similar development with the 
appropriate changes to the reaction (in the case of the dissociative mechanism) or the charge state of the defects.

The kick-out mechanism of $\mathrm{Zn}$ in GaSb is described by the following reaction:

$$
Z n_{I}^{+}+G a^{0} \stackrel{r_{+}, r_{-}}{\longrightarrow} Z n_{G a}^{-}+G a_{I}^{0}+2 h^{+}
$$

Equation 2-15

Interstitial $\mathrm{Zn}$ diffuses as a donor in the singly positive charge state and reacts with a neutral Ga on a substitutional site. Zn "kinks out" the Ga, taking over the substitutional position becoming an ionized acceptor, and resulting in a neutral self-interstitial and two extra holes. The reaction is reversible, with forward and reverse reaction rates given by $r_{+}$and $r_{-}$, respectively.

The large majority of the $\mathrm{Zn}$ atoms are present in the substitutional position. Only a small percentage of the $\mathrm{Zn}$ is interstitial, and therefore mobile. As the $\mathrm{Zn}$ diffuses in from the surface, an electric field is set up by the ionized $\mathrm{Zn}$ acceptors on substitutional sites. The electric field has an impact on the charged mobile species (the electric field effect is of no consequence to neutral Ga self-interstitials), thus altering the flux of atomic species.

Charge effects add to the random walk processes described by Fick's Equation. A directional drift velocity term must be added to describe the flux $J$ of an ionized species. The drift velocity $v_{d}$ is defined as

$$
v_{d}=Z q E=\mu \vec{E}
$$

Equation 2-16

where $Z$ is the ionic valence, $q$ is the electronic charge, $E$ is the electric field, and $\mu$ is the mobility. The added velocity to the charged species will give a second component to the flux relation in addition to the random walk: 


$$
J=-D \frac{\partial C}{\partial x}+\mu C \vec{E}(x)
$$

The electric field can be expressed as the derivative of the potential $\psi$

$$
\vec{E}(x)=-\frac{\partial \psi}{\partial x}
$$

Using the derivative of the local free electron concentration

$$
\frac{\partial n(x)}{\partial x}=N_{C} \exp \left(\frac{E_{F}-E_{C}+e \psi(x)}{k T}\right) \frac{e \frac{\partial \psi}{\partial x}}{k T}
$$

the electric field can be rewritten as

$$
\vec{E}(x)=-\frac{k T}{e} \frac{1}{n(x)} \frac{\partial n(x)}{\partial x} .
$$

Equation 2-20

The first term can be replaced using the Einstein relation

$$
\frac{k T}{e}=\frac{Z D}{\mu}
$$

allowing Equation 2-17 to be rewritten as

$$
J=-D \frac{\partial C}{\partial x}-Z C D \frac{1}{n(x)} \frac{\partial n(x)}{\partial x}
$$

Equation 2-22

Since the semiconductor is being doped p-type, the hole concentration dominates. Furthermore, the term $Q$ is added, allowing for formation or removal of species through chemical reaction, giving a final form of the equation as the following:

$$
J=-D \frac{\partial C}{\partial x}-Z C D \frac{1}{p(x)} \frac{\partial p(x)}{\partial x}+Q .
$$

Equation 2-23

In order to completely describe $\mathrm{Zn}$ diffusion via the kick-out mechanism, each non-matrix component of the reaction (Equation 2-15) must be accounted for with its 
own relation using the modified Fick's Equation. In the following equations, $Z n_{G a}$ refers to substitutional $\mathrm{Zn}$ (always singly negatively charged), $Z n_{I}$ refers to interstitial $\mathrm{Zn}$ (for the purpose of this example, always singly positively charged), $G a$ refers to substitutional $\mathrm{Ga}$ (always neutral), and $G a_{I}$ refers to self-interstitials (for this example always neutral).

$$
\begin{gathered}
\frac{\partial C_{Z n_{G a}}}{\partial t}=r_{+} C_{Z n_{I}} C_{G a}-r_{-} C_{Z n_{G a}} C_{G a_{I}} p^{2} \\
\frac{\partial C_{Z n_{I}}}{\partial t}=\frac{\partial}{\partial x}\left(D_{Z n_{I}} \frac{\partial C_{Z n_{I}}}{\partial x}-\frac{C_{Z n_{I}} D_{Z n_{I}}}{p} \frac{\partial p}{\partial x}\right)-r_{+} C_{Z n_{I}} C_{G a}+r_{-} C_{Z n_{G a}} C_{G a_{I}} p^{2} \\
\frac{\partial C_{G a_{I}}}{\partial t}=\frac{\partial}{\partial x}\left(D_{G a_{I}} \frac{\partial C_{G a_{I}}}{\partial x}\right)+r_{+} C_{Z n_{I}} C_{G a}-r_{-} C_{Z n_{G a}} C_{G a_{I}} p^{2}
\end{gathered}
$$

Normalized variables, defined below, can be introduced to make the doping dependence implicit within the calculations, making them simpler.

$$
\begin{gathered}
\widetilde{C}_{Z n_{G a}} \equiv \frac{C_{Z n_{G a}}}{C_{Z n_{G a}}^{e q}} \\
\widetilde{C}_{Z n_{I}} \equiv \frac{C_{Z n_{I}}}{C_{Z n_{I}}^{e q}} \\
\widetilde{C}_{G a_{I}} \equiv \frac{C_{G a_{I}}}{C_{G a_{I}}^{e q}} \\
\widetilde{n}_{i} \equiv \frac{n_{i}}{C_{Z n_{G a}}^{e q}} \\
\widetilde{p} \equiv \frac{p}{p_{e q}}
\end{gathered}
$$

A further simplification can be carried out by imposing chemical equilibrium on the reaction, described by 


$$
\frac{r_{+}}{r_{-}}=\frac{C_{Z n_{G a}}^{e q} C_{G a_{I}}^{e q}}{C_{Z n_{I}}^{e q} C_{G a}} p^{2}
$$

An accurate description of the p-doping is also required which takes into account the compensation of the $\mathrm{Zn}$ acceptors by the interstitials:

$$
p_{e q}^{2}=\frac{1}{2}\left[\left(C_{Z n_{G a}}-C_{Z n_{I}}\right)+\sqrt{\left(C_{Z n_{G a}}-C_{Z n_{I}}\right)^{2}+4 n_{i}^{2}}\right]
$$

Using Equations 2-25 through 2-33, a set of three coupled differential equations can be written down describing the kick-out mechanism.

$$
\left.\begin{array}{c}
\frac{\partial \widetilde{C}_{Z n_{G a}}}{\partial t}=r_{-} C_{G a_{I}}^{e q}\left(\widetilde{C}_{Z n_{I}}-\widetilde{C}_{Z n_{G a}} \widetilde{C}_{G a_{I}} \widetilde{p}^{2}\right) \\
\frac{C_{Z n_{I}}^{e q}}{C_{Z n_{G a}}^{e q}} \frac{\partial \widetilde{C}_{Z n_{I}}}{\partial t}+\frac{\partial}{\partial x}\left(\frac{\partial\left(\widetilde{C}_{Z n_{G a}}-\frac{C_{Z n_{I}}^{e q}}{C_{Z n_{G a}}^{e q}} \widetilde{C}_{Z n_{I}}\right)}{\partial x}\right) \\
\widetilde{C}_{Z n_{I}}\left(\frac{C_{Z n_{I}}^{e q} D_{Z n_{I}}}{C_{Z n_{G a}}^{e q}} \frac{\text { Equation 2-34 }}{\sqrt{\left(\widetilde{C}_{Z n_{G a}}-\frac{C_{Z n_{I}}^{e q}}{C_{Z n_{G a}}^{e q}} \widetilde{C}_{Z n_{I}}\right)^{2}+4 \widetilde{n}_{i}^{2}}}-\frac{C_{Z n_{I}}^{e q} D_{Z n_{I}}}{C_{Z n_{G a}}^{e q}} \frac{\partial \widetilde{C}_{Z n_{I}}}{\partial x}\right)=-r_{-} C_{G a_{I}}^{e q}\left(\widetilde{C}_{Z n_{I}}-\widetilde{C}_{Z n_{G a}} \widetilde{C}_{G a_{I}} \widetilde{p}^{2}\right) \\
\end{array}\right)=
$$

Equation 2-35

$$
\frac{C_{G a_{I}}^{e q}}{C_{Z n_{G a}}^{e q}} \frac{\partial \widetilde{C}_{G a_{I}}}{\partial t}+\frac{\partial}{\partial x}\left(-\frac{C_{G a_{I}}^{e q} D_{G a_{I}}}{C_{Z n_{G a}}^{e q}} \frac{\partial \widetilde{C}_{G a_{I}}}{\partial x}\right)=r_{-} C_{G a_{I}}^{e q}\left(\widetilde{C}_{Z n_{I}}-\widetilde{C}_{Z n_{G a}} \widetilde{C}_{G a_{I}} \tilde{p}^{2}\right)
$$

This completes the set of coupled partial differential equations (CPDE) which are used to numerically fit the $\mathrm{Zn}$ diffusion profile in GaSb. As stated at the beginning, the example developed was for the case of $\mathrm{Zn}$ diffusion proceeding via the kick-out mechanism, mediated by neutral Ga self-interstitials. Changing the charge state of the self-interstitials alters the diffusion behavior due to the electric field imposed by the 
substitutional Zn. Changing the diffusion mechanism also results in a change of the CPDEs since a different reaction describes the diffusion.

As was discussed in Section 1.4.2, no clear identification of the mechanism of $\mathrm{Zn}$ diffusion in GaSb has been made. Previous studies have indicated that a substitutionalinterstitial diffusion mechanism is active, referring to either the kick-out, or the dissociative mechanism. Using the development outlined above, predictive forms of the diffusion profiles can be calculated for the two possible diffusion mechanisms. Figure 2-12 and Figure 2-13 show the predicted shape of the diffusion profiles of $\mathrm{Zn}$ in $\mathrm{GaSb}$ diffusing from a constant surface concentration for the kick-out and the dissociative mechanism respectively. From the figures it is apparent that very distinctive profiles develop for different charge states and mechanisms. 


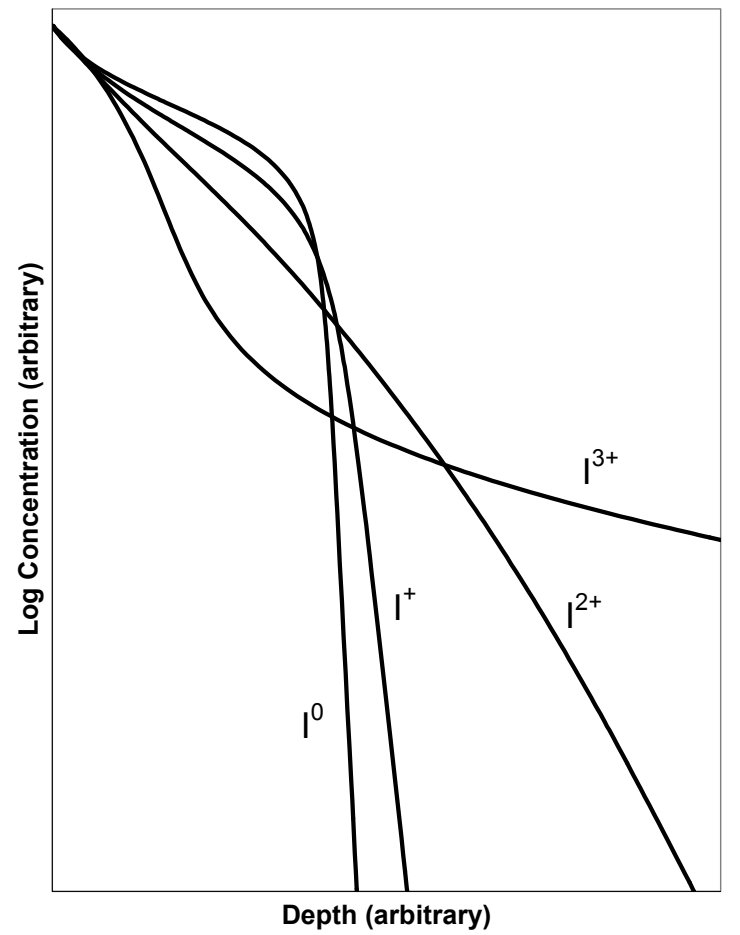

Figure 2-12: Expected profile for impurity diffusion via the kick-out mechanism mediated by native intersititals of various charge types.

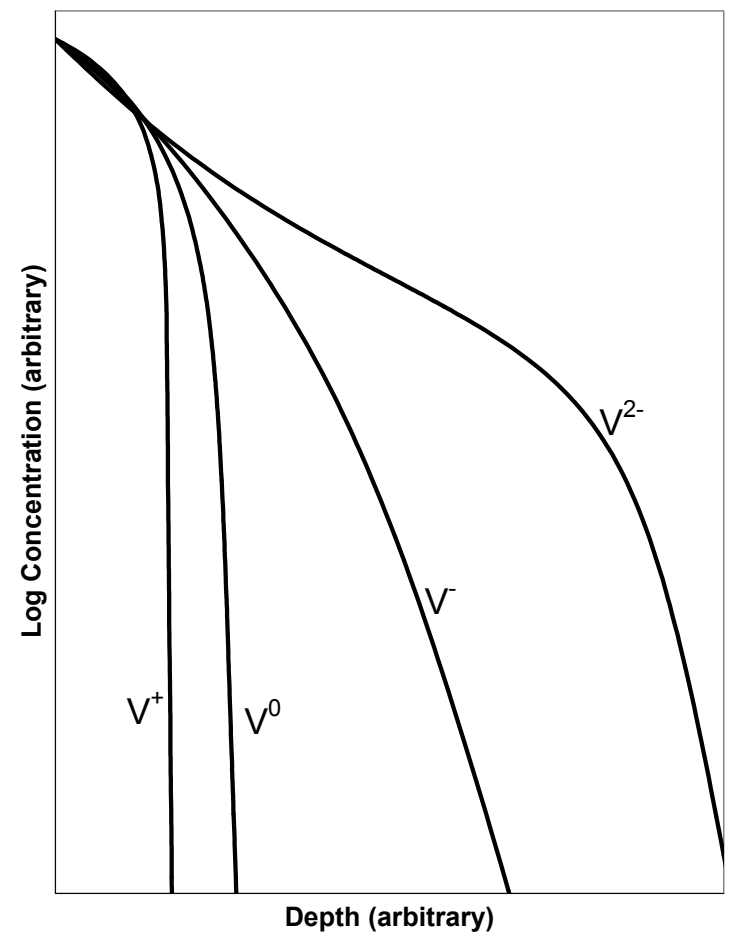

Figure 2-13: Expected profile for impurity diffusion via the dissociative mechanism mediated by native vacancies of various charge types.

While the figures above do not provide any quantitative results, they do allow for qualitative analysis of the $\mathrm{Zn}$ diffusion profiles. 


\section{Experimental}

\subsection{Sample Structures}

\subsubsection{Isotopic Heterostructures}

Prof. Fernando Briones of the Instituto de Microelectrónica de Madrid, part of the Centro Nacional de Microelectrónica, grew the isotopic heterostructures through molecular beam epitaxy (MBE). The substrates used for growth were from a $50.5 \mathrm{~mm}$ diameter undoped (100) GaSb wafer. MCP Wafer Technology Ltd. of the UK reported a majority carrier concentration (p-type) of $1.3 \times 10^{17} \mathrm{~cm}^{-3}$. The substrates were attached to the holder using a tungsten clip which left a shadow mark with the appearance of a large scratch on the growth surface. The clip was used instead of the preferred molten indium because indium reacts with GaSb. The samples were heated by direct radiation from a filament onto the backsides of the GaSb substrates to a growth temperature of $450^{\circ} \mathrm{C}$. The substrate was diced into approximately $1-2 \mathrm{~cm}^{2}$ sized pieces.

Layers were grown using a valved six-cell MBE setup. The isotopically enriched sources purchased by our group from Russian sources were of unknown purity. They were delivered in the form of small, apparently oxide-free ingots, protected by sealed glass ampoules. A buffer layer was first grown on the substrate using cells of $\mathrm{Ga}$ and $\mathrm{Sb}$ of natural isotopic composition. Enriched layers were grown using cells of enriched ${ }^{69} \mathrm{Ga},{ }^{71} \mathrm{Ga},{ }^{121} \mathrm{Sb}$ and ${ }^{123} \mathrm{Sb}$. On top of the enriched layers, a natural composition cap of GaSb was grown. In order to minimize knock-on effects (discussed in Section 7.1), the first layer was grown using the heavier isotopes $\left({ }^{71} \mathrm{Ga}\right.$ and $\left.{ }^{123} \mathrm{Sb}\right)$. A schematic of a sample structure is shown below. 


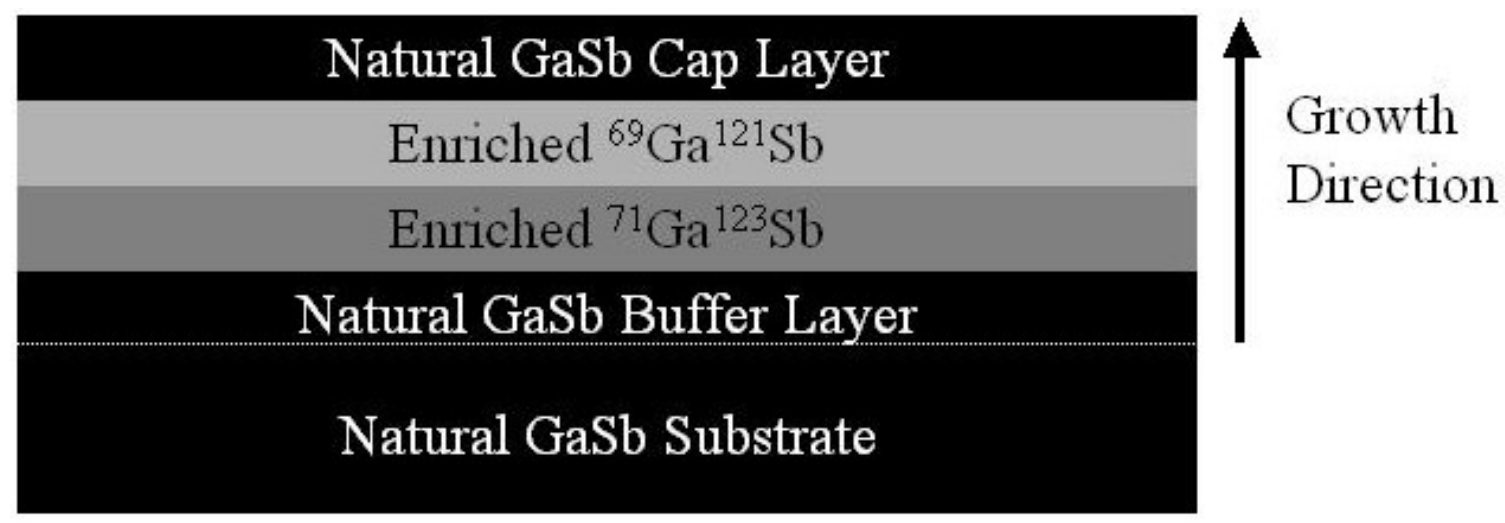

Figure 3-1: Schematic of as-grown GaSb isotopic heterostructure.

\subsubsection{Bulk GaSb}

For the $\mathrm{Zn}$ diffusion into bulk GaSb, $50.8 \mathrm{~mm}$ diameter undoped (100) GaSb wafers with a free carrier concentration of $1-2 \times 10^{16} \mathrm{~cm}^{-3}$ (p-type) were obtained from Atomergic Chemetals Corp.

\subsection{Sample Processing}

Sample preparation for both the isotopic heterostructures used for self-diffusion and for the bulk GaSb used for the $\mathrm{Zn}$ diffusion experiments was identical. The samples were cleaned in organic solvents, first in boiling xylenes for 5 minutes, followed by rinsing and soaking in warm acetone, and finally rinsing with methanol. The samples were then soaked in $\mathrm{HCl}$ for 30 s followed by 10 s etching in $10 \% \mathrm{HF}$.

Diffusion was accomplished using the sealed ampoule technique. Ampoules were made from semiconductor grade quartz tubing purchased from GM Associates, Inc. Prior to using an ampoule, both the shell and plug were cleaned with acetone and etched in $10 \% \mathrm{HF}$ for one minute.

Samples and any dopant diffusion source were placed in the ampoule. With the plug in place but not affixed to the ampoule shell, the ampoule was mounted to the 
ampoule sealing station. The sealing station allows vacuum to be drawn on ampoules by a turbo pump backed up by a roughing pump. Inert gases can also be added to the ampoule prior to sealing. After pulling a rough vacuum, the ampoules were heated with a hydrogen flame to evaporate any solvent remnants. The ampoules were then flushed 5 times with Ar and subsequently evacuated to remove any trace solvent vapor. Prior to sealing the ampoule with a hydrogen-oxygen torch, its base pressure was reduced to 1$2 \times 10^{-5}$ torr as measured by an ion gauge.

The sealed ampoules were annealed in a Lindberg Blue resistively-heated furnace where the temperature can be controlled to $\pm 2 \mathrm{~K}$. Temperature was measured using a Stype thermocouple which was laid inside the furnace, protected by a $1 \mathrm{~mm}$ thick quartz tube. This ampoule was placed in direct contact with the thermocouple tube to assure accurate measurement of the sample temperature within the furnace. To terminate the annealing, the ampoules were quenched in water at room temperature.

\subsubsection{Self-Diffusion}

Prior to annealing, isotopic heterostructure samples were diced into $1 \times 3 \mathrm{~mm}^{2}$ pieces as a compromise for the number of samples and minimum sample size for SIMS measurements. Samples were placed on a cleaned and etched bulk GaSb carrier wafer.

For the $\mathrm{Sb}$ and $\mathrm{Ga}$ rich experiments, $30 \mathrm{mg}$ of $\mathrm{Ga}_{0.40} \mathrm{Sb}_{0.6}$ or $\mathrm{Ga}_{0.65} \mathrm{Sb}_{0.35}$ respectively was added to the ampoule prior to sealing. The anneals were carried out at a constant temperature with experiments covering a range in temperatures from 571 to $708^{\circ} \mathrm{C}$. 
Due to the size of the isotopic heterostructure samples, the samples could not be loaded directly into the SIMS instrument sample holder. The samples were glued to Ge block holders using silver paint to avoid charging of the sample during analysis (see Section 7.1). The blocks were cut from a Ge ingot using an inner diameter circular diamond saw. They were designed with a groove of the same dimensions as the sample to be analyzed, particularly in the height, to maintain a flat surface across the sample to the block. This was done to minimize any edge effects during SIMS sputtering. A diagram of the blocks used can be seen below.

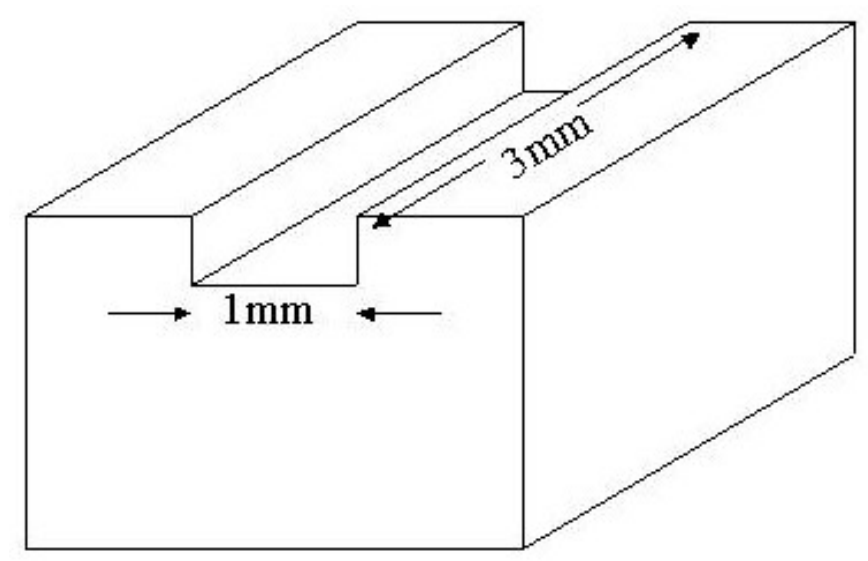

Figure 3-2: Ge block used for SIMS analysis of isotopic structures.

\subsection{2 $\underline{\text { Zn Diffusion }}$}

$4 \times 8 \mathrm{~mm}^{2}$ samples were diced from the bulk GaSb wafer described in Section 3.1.2 for these diffusion studies. Approximately $5 \mathrm{mg}$ of $\mathrm{Zn}-\mathrm{Ga}$ alloy (see Section 3.3), which had been etched in $\mathrm{HCl}$ for $30 \mathrm{~s}$, was added to the ampoule. Care was taken to have the diffusion source be free of contact to the GaSb sample to assure indiffusion of $\mathrm{Zn}$ into GaSb via the gaseous phase. 


\subsection{Alloy Source Preparation}

A solid solution alloy of $\mathrm{Zn}$ and $\mathrm{Ga}$ was used for all $\mathrm{Zn}$ diffusion experiments to create a $\mathrm{Zn}$ atmosphere over the GaSb in the ampoule. The use of elemental $\mathrm{Zn}$ at the desired diffusion temperatures would result in $\mathrm{Zn}$ surface concentrations far exceeding the desired doping levels. By using diluted $\mathrm{Zn}-\mathrm{Ga}$ solid sources, a lower $\mathrm{Zn}$ partial pressure can be achieved in a relatively reproducible manner.

The alloys were prepared by combining known amounts of 7N Ga $(99.99999 \%$ pure) with $6 \mathrm{~N} \mathrm{Zn} \mathrm{(99.9999 \%} \mathrm{pure)} \mathrm{in} \mathrm{an} \mathrm{evacuated} \mathrm{and} \mathrm{sealed} \mathrm{ampoule} \mathrm{prepared} \mathrm{as}$ described in Section 3.2. The ampoule was placed in a vertically mounted, resistively heated furnace at $700^{\circ} \mathrm{C}$ for $24-48$ hours, allowing the $\mathrm{Zn}$ and $\mathrm{Ga}$ to fully homogenize in the liquid form. The samples were quenched in liquid ethylene glycol at $-5^{\circ} \mathrm{C}$ to obtain maximum homogeneity of the solid source. The alloys were stored in the refrigerator to minimize phase separation.

\subsection{SIMS and Calibration}

SIMS concentration profiles of the isotopes of interest were measured by a Cameca IMS-3f by Dr. Jon Erickson of Accurel Systems in Sunnyvale, California. A Cs ${ }^{+}$ ion beam with an energy of approximately $2 \mathrm{keV}$ was used for all profiles.

As discussed in Section 7.1, SIMS measures secondary ions which need to be converted to atomic concentrations. Two different techniques were used for the conversion of the species of interest.

Calibration of each isotope of $\mathrm{Ga}$ and $\mathrm{Sb}$ was accomplished through the known atomic density and isotopic composition of $\mathrm{GaSb}$. The lattice parameter of $\mathrm{GaSb}$ has been measured by x-ray powder diffraction to be $6.09593 \AA$ [M.E. Straumanis, 1965]. 
This value corresponds to an atomic density of $1.766 \times 10^{22} \mathrm{~cm}^{-3}$ for each element. Knowing the natural abundance of the stable isotopes of $\mathrm{Ga}$ and $\mathrm{Sb}$, the atomic densities of the four isotopes can be calculated, as is shown in Table 3-1. These values can be used to correlate secondary ion counts in a natural composition layer to atomic concentration over a whole sample. All samples had areas of natural isotopic composition for this calibration.

\begin{tabular}{c|c|c|c} 
Element & Isotope & Natural Abundance (\%) & Atomic Density $\left(\mathbf{c m}^{\mathbf{3}}\right)$ \\
\hline \multirow{2}{*}{$\mathbf{G a}$} & ${ }^{{ }^{\mathbf{9}} \mathbf{G a}}$ & 60.11 & $1.061 \times 10^{22}$ \\
& ${ }^{71} \mathbf{G a}$ & 39.89 & $0.7044 \times 10^{22}$ \\
\hline \multirow{2}{*}{$\mathbf{S b}$} & ${ }^{121} \mathbf{S b}$ & 57.36 & $1.013 \times 10^{22}$ \\
& ${ }^{123} \mathbf{S b}$ & 42.64 & $0.7529 \times 10^{22}$
\end{tabular}

Table 3-1 Isotopic composition and atomic density of GaSb

$\mathrm{Zn}$ calibration was achieved through the use of a $\mathrm{Cz}$-grown $\mathrm{Zn}$ doped $\mathrm{GaSb}$ wafer standard from University Wafer with a bulk $\mathrm{Zn}$ concentration of $1-2 \times 10^{18} \mathrm{~cm}^{-3}$. Conversion factors for secondary counts to $\mathrm{Zn}$ concentration were obtained using identical sputtering conditions at each SIMS session. In all analyses, ${ }^{64} \mathrm{Zn}$ was the isotope measured (48.6\% natural abundance).

\subsection{Transmission Electron Microscopy}

Drs. Mourad Benamara and Zuzana Liliental-Weber carried out cross-sectional TEM studies on a number of the Zn-diffused samples at the National Center for Electron Microscopy (NCEM) at Lawrence Berkeley National Lab (LBNL). The TEM microscope used was a JEOL 200CX operating at an accelerating voltage of $200 \mathrm{kV}$. W. Swider prepared the samples for cross-sectional TEM imaging. 


\section{Results}

\subsection{Self-Diffusion}

Prof. Briones delivered multiple isotopic structures. SIMS analysis carried out on all the samples revealed large variations in the quality of the grown structures (see Section 2.4.2). Sample 2583-1 was used for all diffusion experiments. The SIMS profile of this sample can be seen in Figure 4-1. $150 \mathrm{~nm}$ thick layers of ${ }^{71} \mathrm{Ga}^{123} \mathrm{Sb}$ and ${ }^{69} \mathrm{Ga}^{121} \mathrm{Sb}$, forming the isotopic heterostructure, were grown on a $200 \mathrm{~nm}$ thick buffer layer. A 200 $\mathrm{nm}$ capping layer is also clearly visible at the surface. At the interface of the two isotopically enriched layers one can see a two order of magnitude concentration difference between the Ga and $\mathrm{Sb}$ isotopes, with the $\mathrm{Sb}$ showing a slightly better structure for diffusion studies (see Section 2.4.2). Raman spectroscopy was carried out on the structure by Dr. T. Ruf, resulting in sharp lines consistent with undoped high quality single crystalline GaSb. 


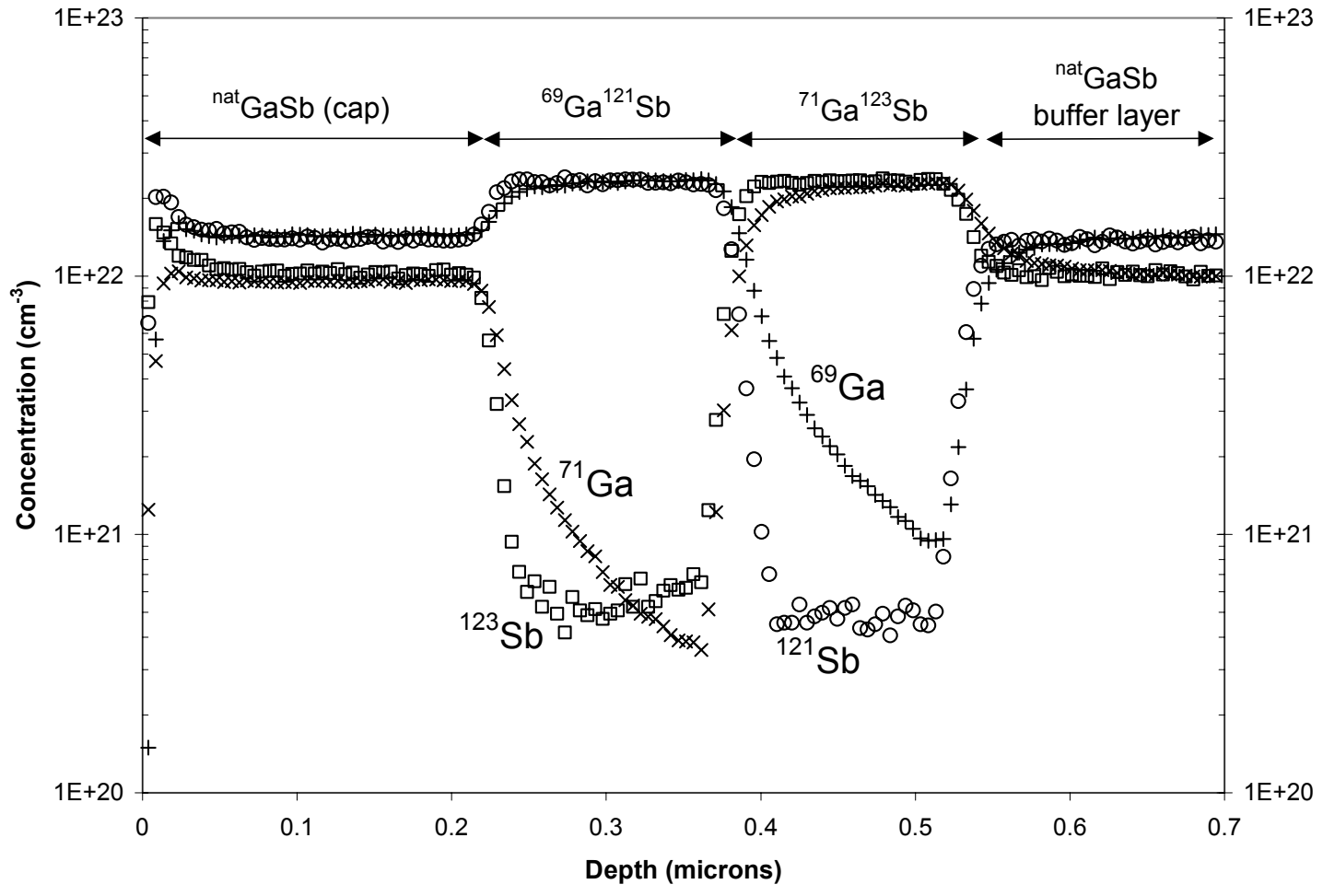

Figure 4-1: SIMS profiles of as-grown isotopic heterostructure used for self-diffusion experiments showing concentrations of ${ }^{69} \mathrm{Ga}(+),{ }^{71} \mathrm{Ga}(\times),{ }^{121} \mathrm{Sb}(\circ)$, and ${ }^{123} \mathrm{Sb}(\square)$. For clarity, only every second data point is plotted.

Initial experiments at low temperature showed significant Ga diffusion, while the $\mathrm{Sb}$ remained stationary. As an example, Figure 4-2 depicts the SIMS concentration profile of the four isotopes following annealing at $590^{\circ} \mathrm{C}$ for 11 days under $\mathrm{Sb}$ rich conditions. Ga diffusion is clearly visible when compared to the as-grown structure, while no change can be detected in the Sb profiles. 


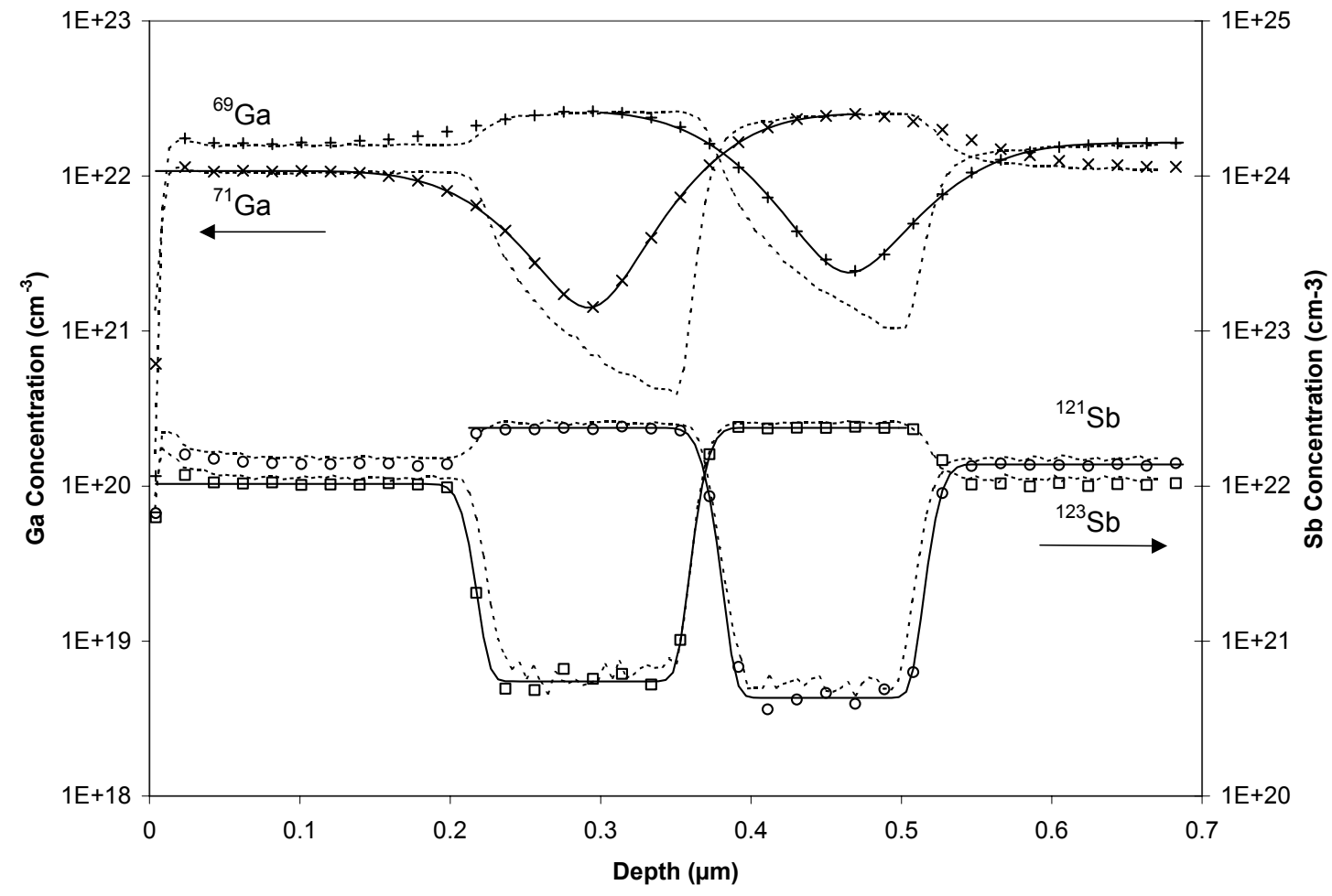

Figure 4-2: SIMS profiles of isotopic heterostructure after annealing at $590^{\circ} \mathrm{C}$ for 11 days under $\mathrm{Sb}$ rich conditions showing concentrations of $69 \mathrm{Ga}(+), 71 \mathrm{Ga}(\times), 121 \mathrm{Sb}(\circ)$, and $123 \mathrm{Sb}(\square)$. For clarity, only every fourth data point is plotted. The thin dashed lines depict the as-grown structure, while the solid lines show the best fits to the diffusion profiles.

This observation of no detectable Sb movement at lower temperatures and short times was observed in all initial experiments. Even annealing at $700^{\circ} \mathrm{C}\left(12^{\circ} \mathrm{C}\right.$ under the melting point of $\mathrm{GaSb}$ ) for 93 hours shows no significant diffusion on the Sb sublattice, while the Ga is nearly completely inter-diffused (see Figure 4-3). 


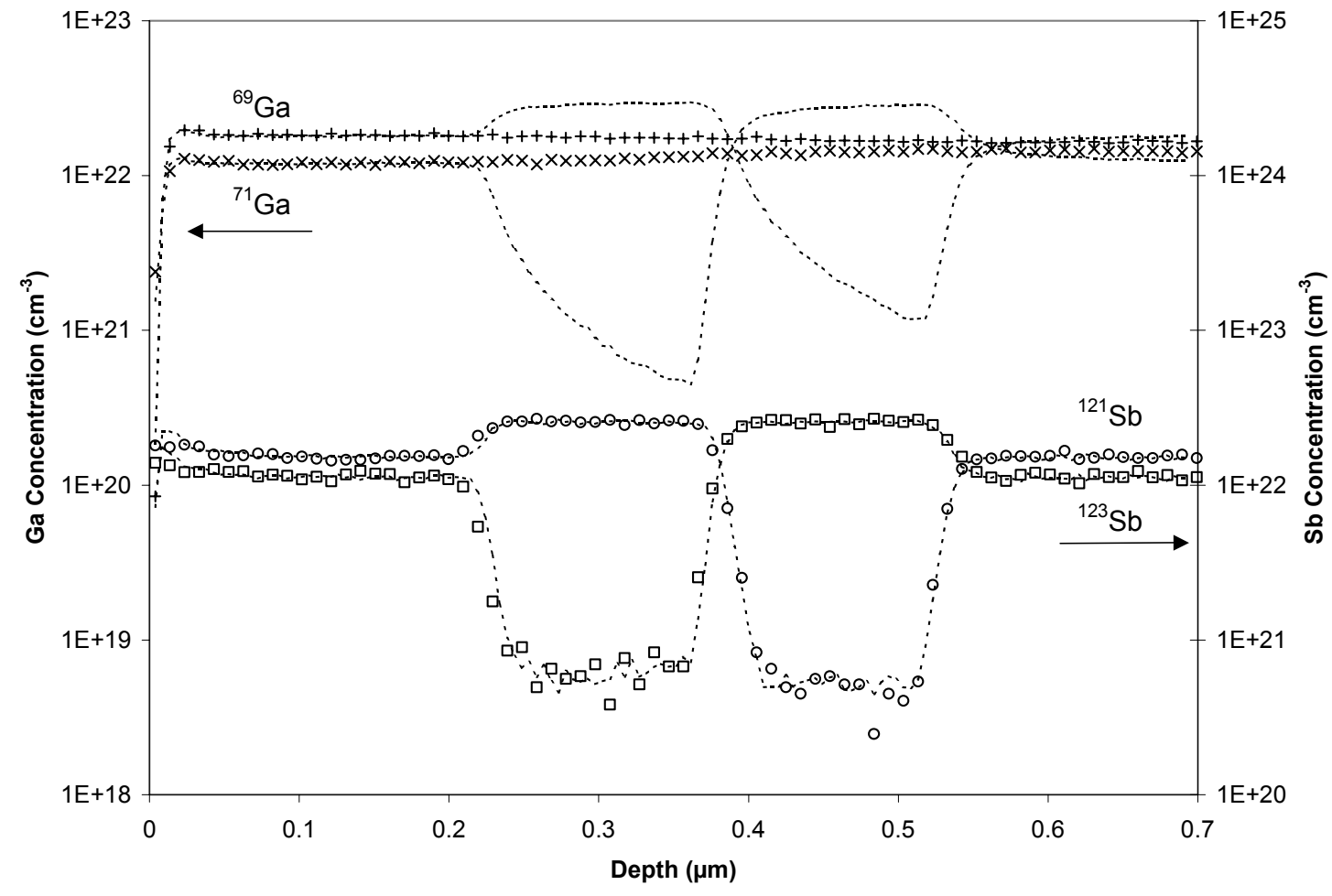

Figure 4-3: SIMS profiles of isotopic heterostructure after annealing at $700^{\circ} \mathrm{C}$ for 93 hours showing concentrations of $69 \mathrm{Ga}(+), 71 \mathrm{Ga}(\times), 121 \mathrm{Sb}(\circ)$, and $123 \mathrm{Sb}(\square)$. The dashed lines indicate the asgrown structure. While the Ga isotopes are nearly completely intermixed, no movement on the Sb sublattice can be observed.

Only through extremely long duration annealing at a temperature just under the melting point of GaSb could Sb diffusion be observed, as depicted in Figure 4-4. After approximately 18 days at $700^{\circ} \mathrm{C}$, the first diffusion at the interface is visible in the form of the rounding of the profiles. Diffusion on the Ga lattice after this anneal has resulted in a completely interdiffused layer. 


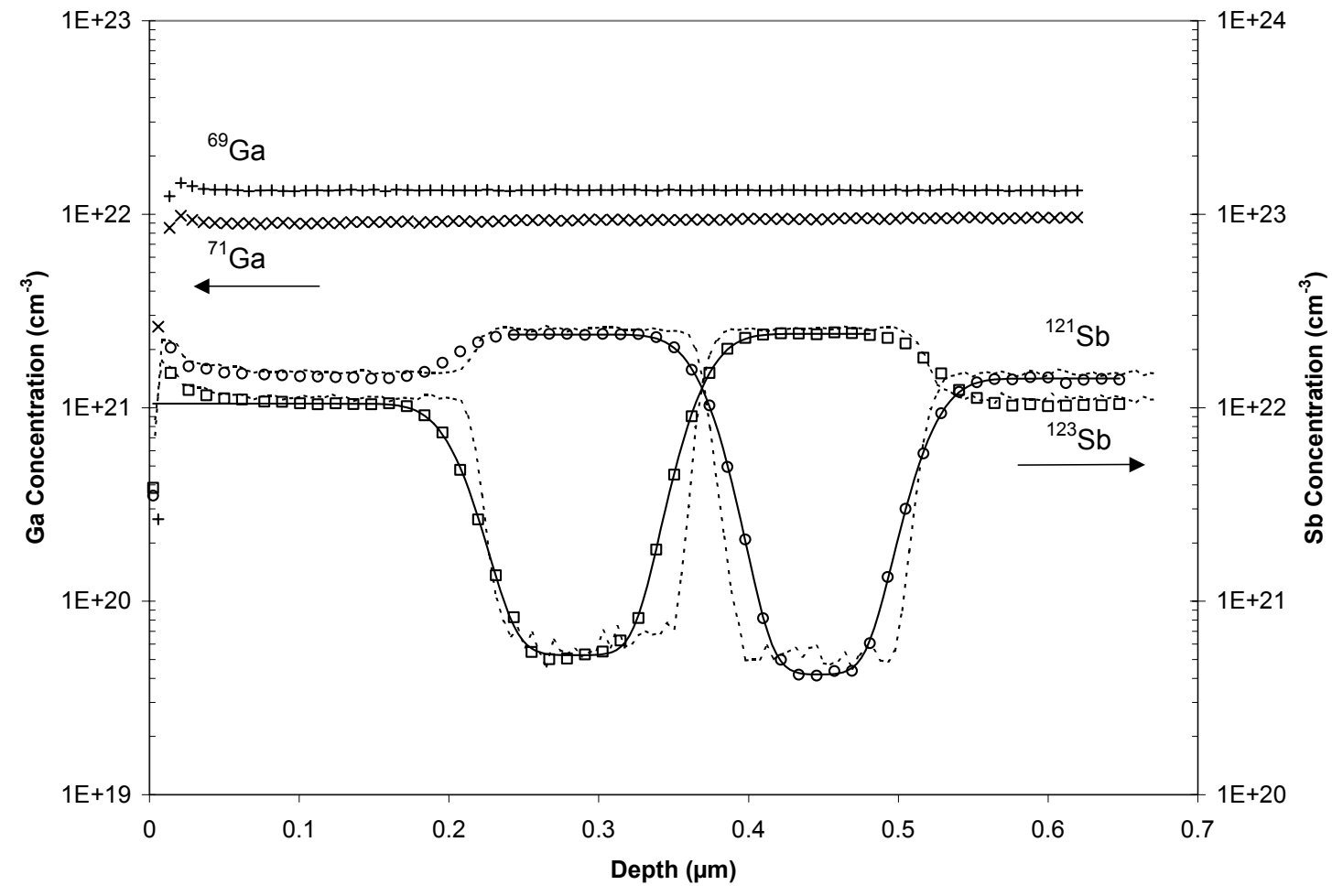

Figure 4-4: SIMS profiles of isotopic heterostructure after annealing at $700^{\circ} \mathrm{C}$ for about 18 days under Sb-rich conditions showing concentrations of ${ }^{69} \mathrm{Ga}(+),{ }^{71} \mathrm{Ga}(\times),{ }^{121} \mathrm{Sb}(\circ)$, and ${ }^{123} \mathrm{Sb}(\square)$. For clarity, only every fourth data point is plotted. The dashed lines indicate the as-grown Sb structure. The solid lines depict the best fits for Sb diffusion while homogenous Ga profiles are clearly visible.

In order to obtain accurate $\mathrm{Sb}$ diffusion data, it became necessary to go to very long anneal times to observe substantial diffusion. Figure 4-5 shows the SIMS profile of the isotopic heterostructure after approximately 85 days at $685^{\circ} \mathrm{C}$. Here, substantial diffusion on the $\mathrm{Sb}$ lattice can be seen for the first time, to a comparable extent of $\mathrm{Ga}$ diffusion observed after 11 days at only $590^{\circ} \mathrm{C}$. 


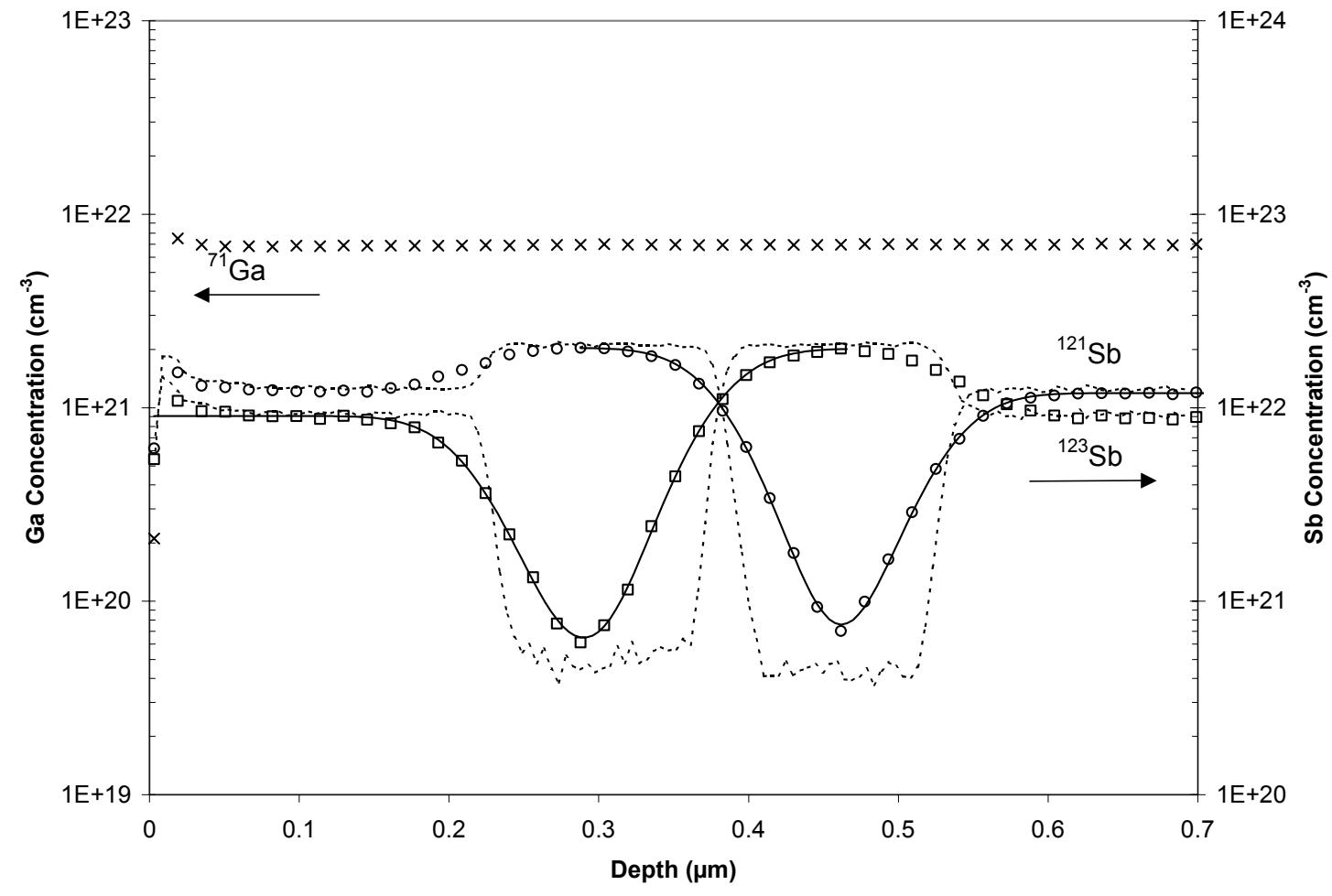

Figure 4-5: SIMS profiles of isotopic heterostructure after annealing at $685^{\circ} \mathrm{C}$ for about 85 days under Sb-rich conditions showing concentrations of ${ }^{71} \mathrm{Ga}(\times),{ }^{121} \mathrm{Sb}(\circ)$, and ${ }^{123} \mathrm{Sb}(\square)$. For clarity, only every fourth data point is plotted. The profile of ${ }^{69} \mathrm{Ga}$ was not measured to increase the number of data points for the Sb fitting. The solid lines depict the best fits for Sb diffusion while a homogenous Ga profile is clearly visible.

The Ga- and Sb-rich annealing conditions had no noticeable effect on the diffusivity of either species within experimental accuracy. An example of this can be seen in Figure 4-6 where the Ga profiles are compared for Ga-rich and Sb-rich conditions after annealing of one day at $642^{\circ} \mathrm{C}$. The $\mathrm{Sb}$ profiles are not shown since they do not differ from the as-grown after this short of an anneal. The high temperature, long time anneals showed similarly no effect on $\mathrm{Sb}$ diffusing in either Ga-rich or Sb-rich environments. 


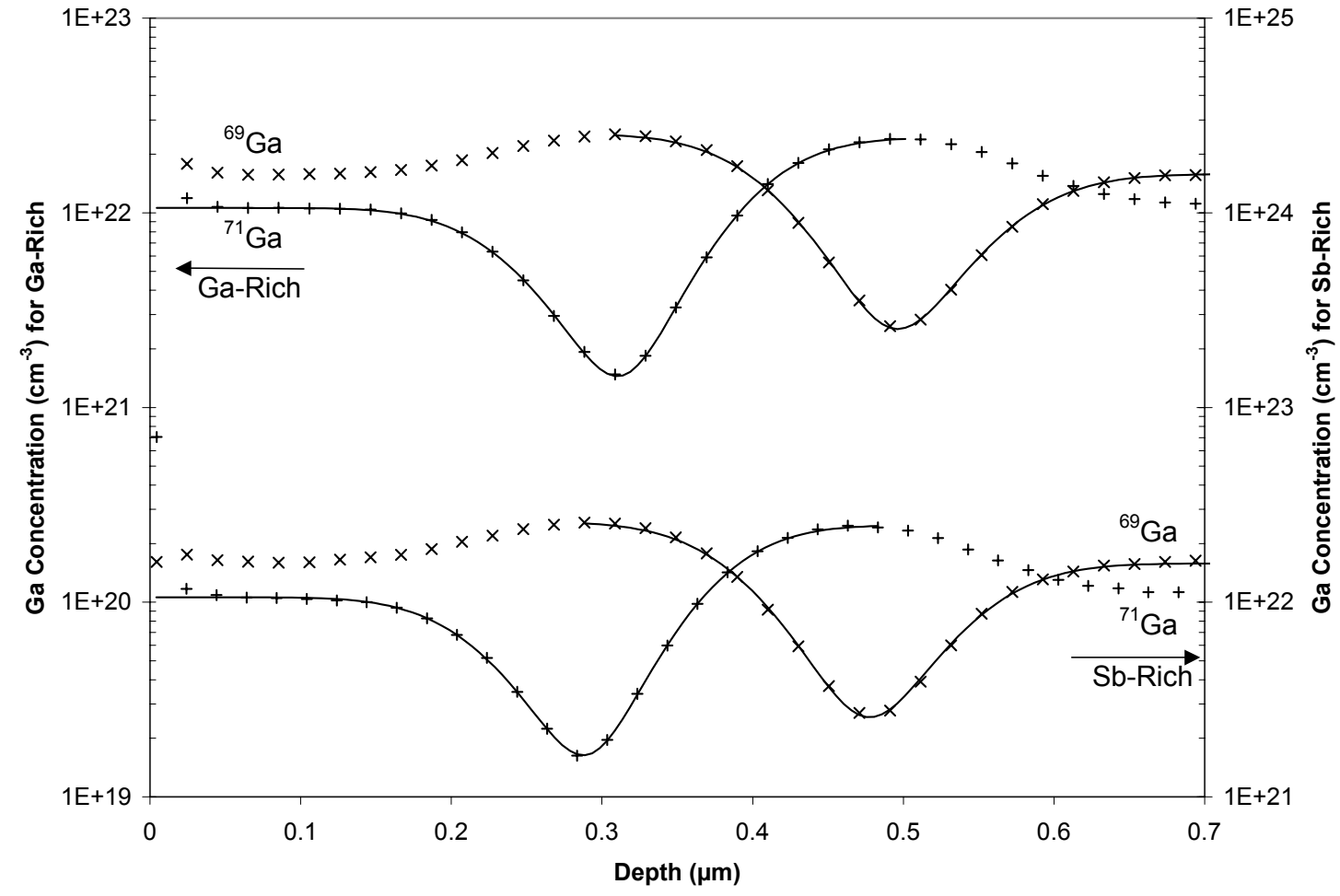

Figure 4-6: SIMS profiles of isotopic heterostructure after annealing at $642^{\circ} \mathrm{C}$ for 1 day under Gaand Sb-rich conditions showing concentrations of ${ }^{69} \mathrm{Ga}(+)$ and ${ }^{71} \mathrm{Ga}(\times)$. For clarity, only every fourth data point is plotted. Ga- and Sb- rich conditions seem to have no effect on the Ga diffusivity. The solid lines depict the best fits for Ga diffusion. The slight difference in hetero-stucture interface depth is attributed to error during the crater depth measurement.

The fitting of the SIMS profiles was achieved by applying Fick's Law for diffusion across an interface (see Section 2.5.1). The solid lines in the previous figures demonstrate the ability of this method to fit the data. The diffusivities of Ga and $\mathrm{Sb}$ were extracted from those profiles showing significant diffusion for the given species, and the results can be seen in Table 4-1. 


\begin{tabular}{|c|c|c|c|c|}
\hline $\mathrm{T}\left({ }^{\circ} \mathrm{C}\right)$ & $\mathrm{t}(\mathrm{s})$ & Ambient & $\mathrm{D}_{\mathrm{Ga}}\left(\mathrm{cm}^{2} / \mathrm{s}\right)$ & $\mathrm{D}_{\mathrm{Sb}}\left(\mathrm{cm}^{2} / \mathrm{s}\right)$ \\
\hline 571 & 2237400 & Sb-rich & $3.1 \times 10^{-18}$ & \\
\hline 590 & 950400 & Sb-rich & $8.8 \times 10^{-18}$ & \\
\hline 590 & 950400 & Ga-rich & $1.3 \times 10^{-17}$ & \\
\hline 615 & 259200 & Sb-rich & $4.0 \times 10^{-17}$ & \\
\hline 642 & 86400 & Sb-rich & $1.1 \times 10^{-16}$ & \\
\hline 642 & 86400 & Ga-rich & $1.2 \times 10^{-16}$ & \\
\hline 670 & 25200 & Sb-rich & $3.7 \times 10^{-16}$ & \\
\hline 670 & 2325900 & Sb-rich & & $7.0 \times 10^{-19}$ \\
\hline 670 & 2325900 & Sb-rich & & $6.2 \times 10^{-19}$ \\
\hline 685 & 7347600 & Sb-rich & & $8.1 \times 10^{-19}$ \\
\hline 692 & 7347600 & Sb-rich & & $9.6 \times 10^{-19}$ \\
\hline 700 & 6300 & Sb-rich & $1.2 \times 10^{-15}$ & \\
\hline 700 & 6300 & Ga-rich & $8.8 \times 10^{-16}$ & \\
\hline 700 & 334800 & Ga-rich & & $<1.5 \times 10^{-18}$ \\
\hline 700 & 1564500 & Sb-rich & & $1.1 \times 10^{-18}$ \\
\hline 708 & 574200 & Sb-rich & & $1.5 \times 10^{-18}$ \\
\hline
\end{tabular}

Table 4-1.: Ga and Sb diffusion coefficients extracted from self-diffusion experiments.

When the temperature dependencies of the diffusivities are plotted, a clear Arrhenius behavior is observed for both $\mathrm{Ga}$ and $\mathrm{Sb}$. Figure 4-7 shows these results along with the data reported by Weiler and Mehrer (1984). These results for the diffusivity of $\mathrm{Ga}$ are within an order of magnitude of the older results, but the diffusivity of $\mathrm{Sb}$ is over three orders of magnitude below those of Weiler and Mehrer. 


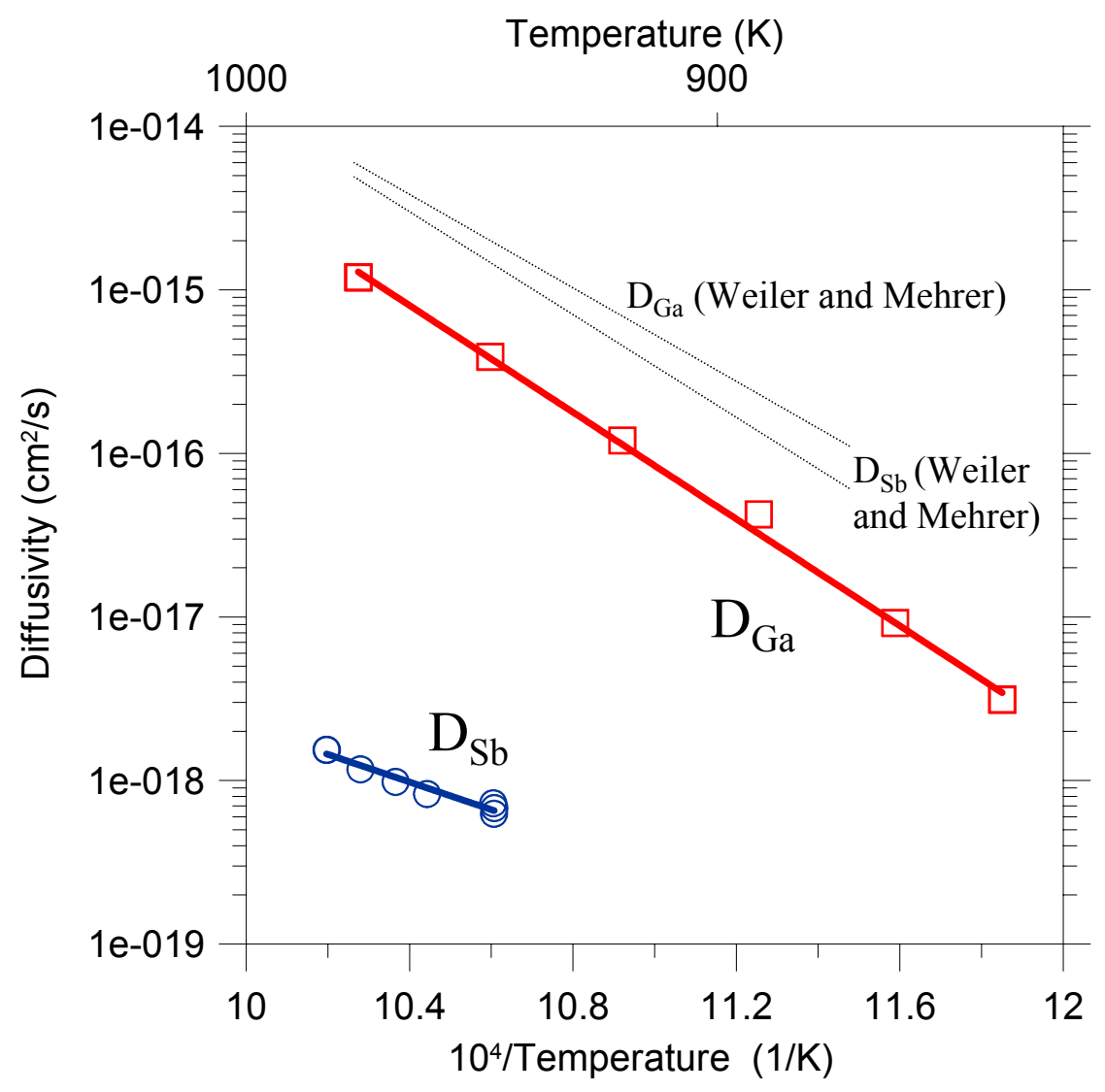

Figure 4-7: The temperature dependencies of the diffusivities of Ga ( $\square$ ) and Sb ( $\odot$ ) under Sb-rich conditions plotted alongside the self-diffusion data from Weiler and Mehrer (1984) depicted as the light dotted lines.

\section{2 $\underline{\text { Zn Diffusion }}$}

SIMS depth concentration profiles of $\mathrm{Zn}$ were obtained showing the two characteristic shapes described in Section 1.4.2. Figure 4-8 shows examples of the kinkand-tail and box type profiles. The kink-and-tail profiles are clearly identifiable by the presence of the double hump-like structure while this characteristic is missing in the box profiles. Kink-and-tail profiles were observed in all samples measured with Zn surface concentrations in excess $10^{20} \mathrm{~cm}^{-3}$ while box profiles were observed in all samples measuring surface concentrations below $10^{20} \mathrm{~cm}^{-3}$. 


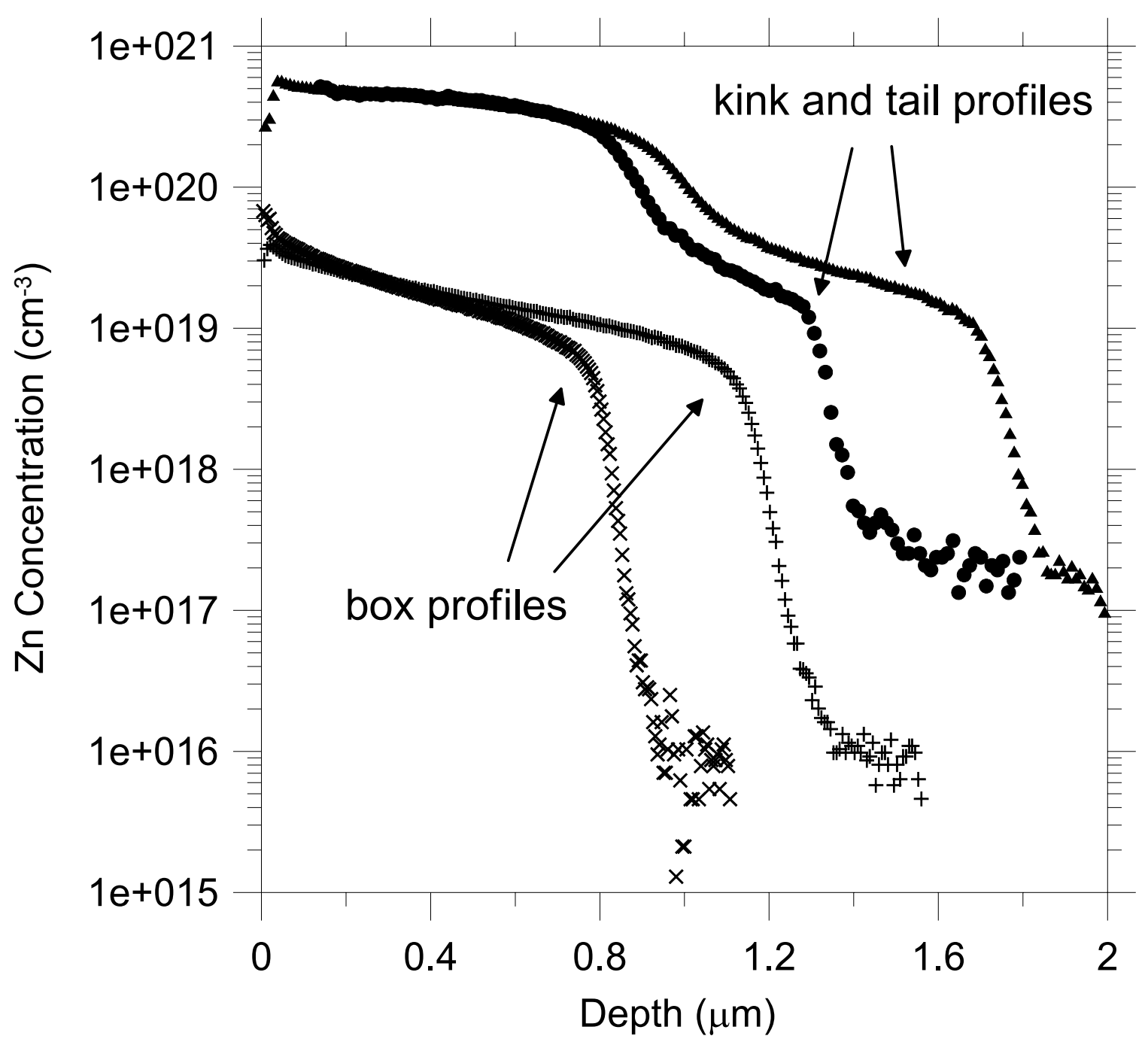

Figure 4-8: Two examples each of kink and tail and box shaped $\mathrm{Zn}$ diffusion profiles in GaSb as measured by SIMS. Annealing schedules and Zn sources are as follows: 4\% Zn / 96\% Ga source, 30 minutes at $640^{\circ} \mathrm{C}(\times) ; 4 \% \mathrm{Zn} / 96 \%$ Ga source, 114 minutes at $638^{\circ} \mathrm{C}(+) ; 20 \% \mathrm{Zn} / 80 \%$ Ga source, 60 minutes, $550^{\circ} \mathrm{C}(\bullet) ; 3 \% \mathrm{Zn} / 39 \% \mathrm{Ga} / 58 \%$ Sb source, 917 minutes at $576^{\circ} \mathrm{C}(\Delta)$.

Cross-sectional TEM was carried out on representative samples showing box and kink-and-tail profiles. Samples showing the box shaped diffusion profiles showed no region of extended defects in the area of diffusion, while the kink-and-tail samples showed a loose network of dislocations behind the diffusion front. An example of the defective samples can be seen in Figure 4-9 where the SIMS profile and TEM are overlaid with correct depth axis for comparison. Two defective regions are clearly visible in the TEM, the first being approximately $450 \mathrm{~nm}$ below the surface, and the 
second near $800 \mathrm{~nm}$. The defect band at $800 \mathrm{~nm}$ is formed by dislocations of the type $1 / 2<110>$. No defective regions were found in any of the samples analyzed with TEM displaying box shaped diffusion profiles, an example of which can be seen in Figure 4-10.

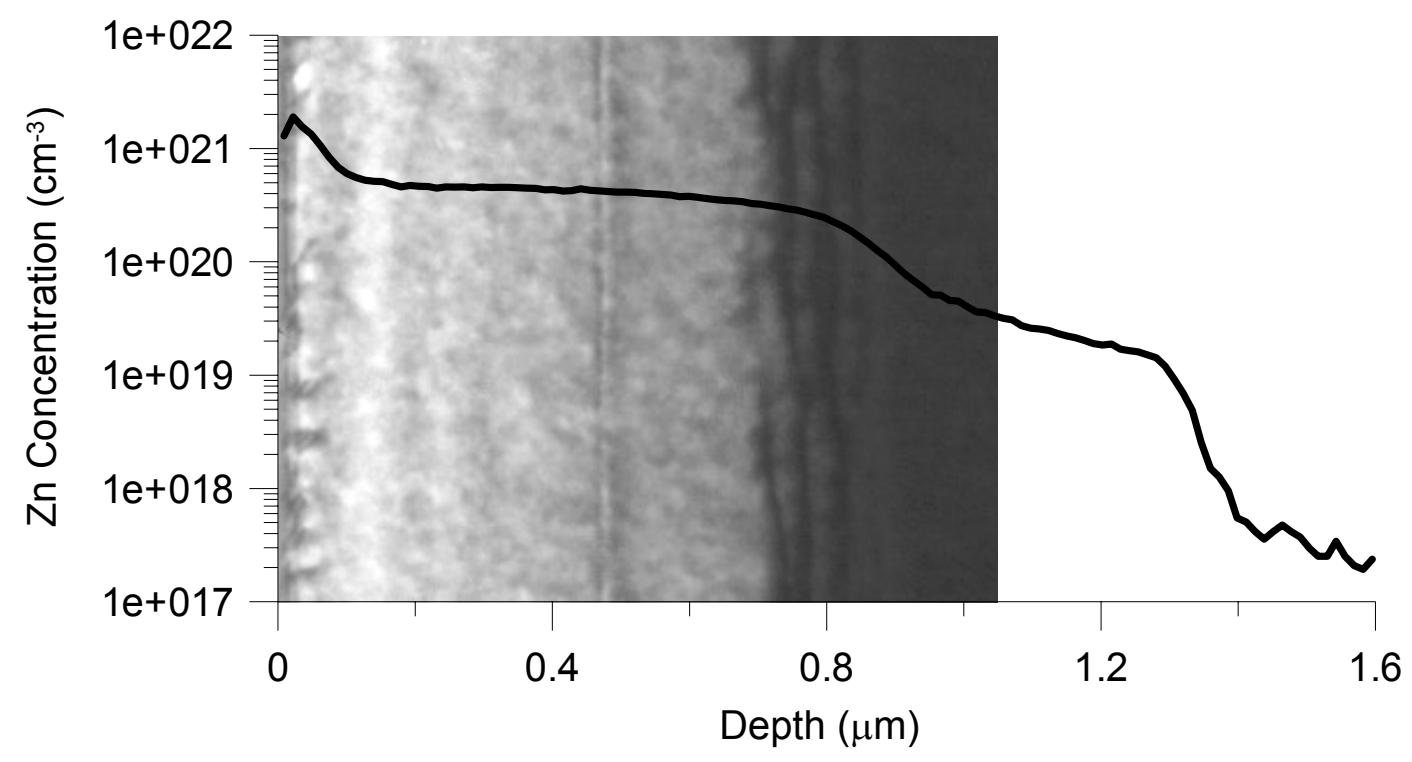

Figure 4-9: Overlaid SIMS and TEM with common depth axis of a sample annealed at $550^{\circ} \mathrm{C}$ for 60 minutes using a $20 \% \mathrm{Zn} / \mathbf{8 0} \%$ Ga source. The SIMS clearly shows a kink-and-tail diffusion profile while the TEM shows two defective regions approximately 450 and $800 \mathrm{~nm}$ from the surface.

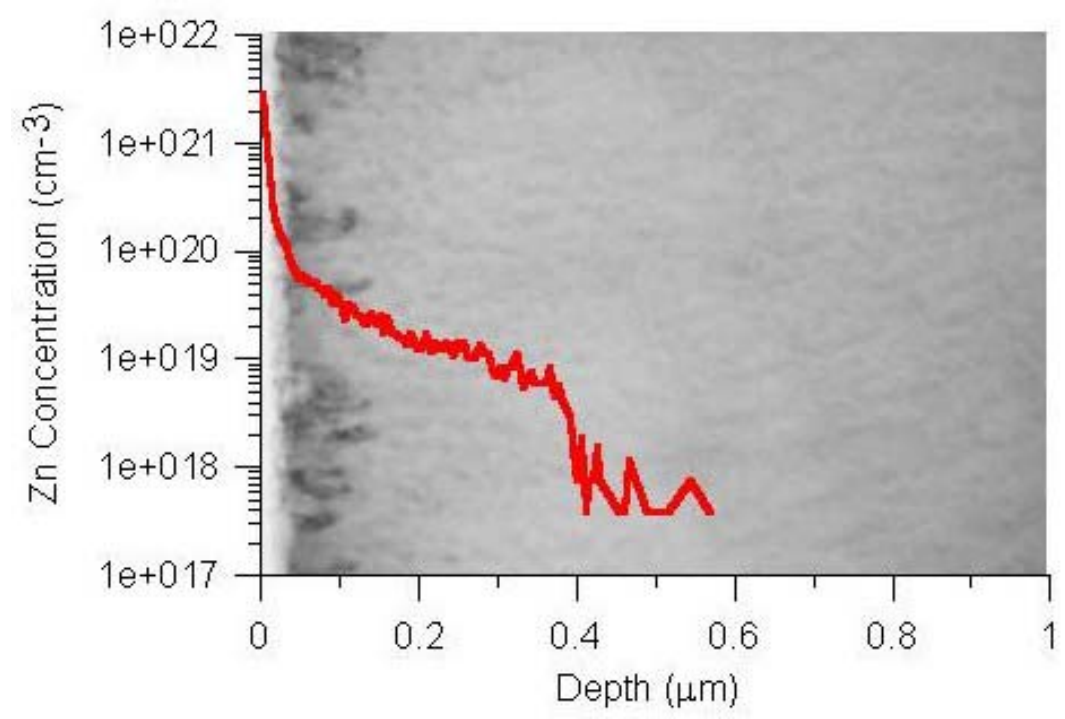

Figure 4-10: Overlaid SIMS and TEM with common depth axis of a sample showing a box-shaped $\mathrm{Zn}$ diffusion profile. No defective regions near the diffusion front can be observed. 


\section{Discussion}

\subsection{Self-Diffusion}

The quality of the as-grown structure was not of ideal nature for the diffusion experiments, but it did suffice. Prof. Briones could not rule out cross-contamination of the layers during growth due to memory effects of the valved MBE cells. As can be readily seen in Figure 4-1, the Ga profiles are distinctly different compared to the $\mathrm{Sb}$ profiles. Seen from the surface, the concentration of the depleted species drops off quickly to approximately two orders of magnitude below the enriched species, but it slowly rises over the subsequent $150 \mathrm{~nm}$ of the layer. Knock-on caused by the $\mathrm{Cs}^{+}$beam during SIMS analysis could have an effect on the broadening of the interface, but this is unlikely, given that the antimony shows no such broadening effect despite the larger atomic mass of $\mathrm{Sb}$.

The most significant result from the self-diffusion experiments is that $\mathrm{Sb}$ diffuses approximately three orders of magnitude more slowly than Ga. The coupled diffusion mechanism proposed by Weiler and Mehrer, based on the similarity in diffusivities, can be directly excluded. The differences between this data and that obtained earlier can most likely be attributed to inaccuracies in their method. As discussed in Section 1.4.1, they used the radiotracer technique to obtain their data for $\mathrm{Ga}$ and $\mathrm{Sb}$ diffusion. It is unclear at this time how they arrived at such high Sb diffusivity values, yet the scattering of their data suggests it could be traced back to difficulties with broadening effects associated with a rough surface and/or with the sputtering technique used to both deposit the radioactive layers and etch the sample after diffusion. Their sputtering setup, in contrast to modern SIMS instruments which count ions only from a small center region of 
the etched crater, counted all sputtered ions. This has the weakness that ions etched from the sidewalls or the surface will appear to originate from a deeper position.

If the coupled diffusion mechanism is invalid, then the two self-atoms must diffuse via distinct mechanisms on their own sublattices. The total diffusivity of each species will be the sum of the contributions of its respective interstitials and vacancies. Ignoring correlation effects, the diffusivity can be stated as

$$
D_{G a, S b}=\left[V_{G a, S b}\right]^{e q} D_{V_{G a, S b}}+\left[G a_{i}, S b_{i}\right]^{e q} D_{G a_{i}, S b_{i}} \quad \text { Equation 5-1 }
$$

$\left[V_{G a}\right]$ and $\left[G a_{i}\right]$ represent the equilibrium concentration of $\mathrm{Ga}$ vacancies and selfinterstitials respectively, while $D_{V_{G a}}$ and $D_{G a_{i}}$ represent their individual diffusivities.

\subsubsection{Diffusion on the Ga Sublattice}

The diffusivity results for Ga obtained at various temperatures can be accurately described by an Arrhenius relation, as shown in Figure 4-7. The curve of best fit allows values for the preexponential factor $D_{0}$ and the activation enthalpy $Q$ to be extracted (see Equation 2-3). The grown heterostructure allowed for Ga diffusivity to be extracted at two locations: the diffusion of ${ }^{71} \mathrm{Ga}$ into the enriched ${ }^{69} \mathrm{Ga}^{121} \mathrm{Sb}$ region, as well as the diffusion of the ${ }^{69} \mathrm{Ga}$ into the enriched ${ }^{71} \mathrm{Ga}^{123} \mathrm{Sb}$ region. The data for the ${ }^{71} \mathrm{Ga}$ diffusion carried out under Sb-rich atmosphere at temperatures between 571 and $700^{\circ} \mathrm{C}$ lead to $\ln \left(D_{0} / \mathrm{cm}^{2} \mathrm{~s}^{-1}\right)=4.41 \pm 1.27$ and $Q=(3.24 \pm 0.10) \mathrm{eV}$. The values extracted using ${ }^{69} \mathrm{Ga}$ diffusion show equivalent results within experimental error of $\ln \left(D_{0} / \mathrm{cm}^{2} \mathrm{~s}^{-1}\right)=4.62 \pm 1.28$ and $Q=(3.25 \pm 0.10) \mathrm{eV}$.

The similarity in the two results indicates that knock-on during SIMS analysis was not a problem. As discussed in Section 7.1, knock-on occurs when a species 
concentration drops off as the ion beam etches into a surface. In these samples, the junction of the isotopically enriched layers has ${ }^{69} \mathrm{Ga}$ on the near-surface side, making it the species susceptible to knock-on. The as-grown structure would display the most pronounced knock-on as it has the steepest drop in concentration. The extracted data for

${ }^{69} \mathrm{Ga}$ diffusion in this study show a slightly higher value, indicating that knock-on does not have a significant influence on our results.

The finding that the ambient has no effect on Ga diffusion leads to an interesting discussion. A Ga-rich ambient should increase the concentrations of Ga self-interstitials and $\mathrm{Sb}$ vacancies, while a $\mathrm{Sb}$-rich ambient would favor $\mathrm{Sb}$ self-interstitials and $\mathrm{Ga}$ vacancies. In order for the diffusivity to be similar in the two ambients, either the contributions of vacancies and self-interstitials to Ga self-diffusion must be of equal magnitude, or the ambient's effect on equilibrium concentrations is marginal.

Mathiot and Edelin found in 1980 that indium (In), an isoelectronic species in $\mathrm{GaSb}$, diffuses faster under a Sb-rich environment than under a Ga-rich one. Their results were interpreted to indicate that Ga vacancies mediate Ga self-diffusion in $\mathrm{GaSb}$ as the ratio of their extracted $D_{I n}$ values to calculated equilibrium vacancy concentration data remained independent of $\mathrm{Sb}$ partial pressure. Using those same calculated vacancy concentration values, a similar comparison of data from this study can be made. The results are shown in Table 5-1. 


\begin{tabular}{|c|c|c|c|}
\hline $\mathrm{T}\left({ }^{\circ} \mathrm{C}\right)$ & $\mathrm{D}_{\mathrm{Ga}}\left(\mathrm{cm}^{2} \mathrm{~s}^{-1}\right)$ & {$\left[\mathrm{V}_{\mathrm{Ga}}{ }^{\mathrm{eq}}\right]$} & $\mathrm{D}_{\mathrm{Ga}} /\left[\mathrm{V}_{\mathrm{Ga}}{ }^{\mathrm{eq}}\right]\left(\mathrm{cm}^{2} \mathrm{~s}^{-1}\right)$ \\
\hline 571 & $3.1 \times 10^{-18}$ & $9.0 \times 10^{-8}$ & $3.4 \times 10^{-11}$ \\
\hline 590 & $8.8 \times 10^{-18}$ & $1.3 \times 10^{-7}$ & $6.8 \times 10^{-11}$ \\
\hline 590 & $1.3 \times 10^{-17}$ & $1.3 \times 10^{-7}$ & $9.6 \times 10^{-11}$ \\
\hline 615 & $4.0 \times 10^{-17}$ & $2.3 \times 10^{-7}$ & $1.8 \times 10^{-10}$ \\
\hline 642 & $1.1 \times 10^{-16}$ & $4.3 \times 10^{-7}$ & $2.7 \times 10^{-10}$ \\
\hline 642 & $1.2 \times 10^{-16}$ & $4.3 \times 10^{-7}$ & $2.7 \times 10^{-10}$ \\
\hline 670 & $3.7 \times 10^{-16}$ & $8.2 \times 10^{-7}$ & $4.5 \times 10^{-10}$ \\
\hline 700 & $1.2 \times 10^{-15}$ & $1.8 \times 10^{-6}$ & $6.5 \times 10^{-10}$ \\
\hline 700 & $8.8 \times 10^{-16}$ & $1.8 \times 10^{-6}$ & $4.9 \times 10^{-10}$ \\
\hline
\end{tabular}

Table 5-1: Ga diffusivity data from this study, equilibrium Ga vacancy concentration data (atomic fraction) calculated from Edelin and Mathiot (1980), and their ratio.

The ratios calculated above under both Ga- and Sb-rich conditions are similar to the dependence found for the diffusivity of In in GaSb under Ga-rich conditions. This would seem to indicate that the Sb-rich conditions used in these experiments did not have the desired effect of creating an Sb-rich environment within the crystal. This could be possible if the GaSb heterostructures were inadvertently grown under Ga-rich conditions. The close similarity between the Ga and In diffusion data in GaSb supports the vacancy mechanism as the mediating mechanism of diffusion on the Ga sublattice. As shall be discussed in the following section, this result is at odds with the $\mathrm{Zn}$ diffusion results which indicate that Ga self-interstitials mediate diffusion via the kick-out mechanism.

\subsubsection{Diffusion on the Sb Sublattice}

Similarly to the Ga diffusion data, $\mathrm{Sb}$ diffusivity data were extracted from both ${ }^{121} \mathrm{Sb}$ diffusing into the ${ }^{71} \mathrm{Ga}{ }^{123} \mathrm{Sb}$ region, as well as ${ }^{123} \mathrm{Sb}$ diffusion into the ${ }^{69} \mathrm{Ga}^{121} \mathrm{Sb}$ region at temperatures ranging from 670 to $708^{\circ} \mathrm{C}$. Again the results show no significant 
difference between the two in terms of pre-exponential factor or activation enthalpy. For ${ }^{121}$ Sb diffusion, $\ln \left(D_{0} / \mathrm{cm}^{2} \mathrm{~s}^{-1}\right)=-22.3 \pm 2.0$ and $Q=(1.59 \pm 0.17) \mathrm{eV}$ best fit the diffusion data, while for ${ }^{123} \mathrm{Sb}$ diffusion, $\ln \left(D_{0} / \mathrm{cm}^{2} \mathrm{~s}^{-1}\right)=-19.7 \pm 2.8$ and $Q=(1.81 \pm 0.23) \mathrm{eV}$ yields the best fit. The larger errors associated with these values are a result of the smaller number of samples carried out and the narrow temperature range used due to the extremely low diffusivity of $\mathrm{Sb}$.

When a reasonable jump frequency and distance are used in Equation 2-3, the resulting entropy has a negative value. This would indicate that the concentration of the native defect mediating $\mathrm{Sb}$ diffusion decreases with increasing temperature near the melting point, resulting in lower entropy. This type of behavior is not consistent with vacancies, leaving the Sb self-interstitial as the most likely possibility to have this type of behavior near the melting point.

In order for $\mathrm{Sb}$ self-interstitials to be promoted, an Sb-rich crystal must be present. As previously discussed for Ga diffusion, it is believed that the isotopic heterostructure was grown on the Ga-rich side of the phase diagram and that this condition dominates during the Ga diffusion, both under Ga- and Sb-rich ambients. However, no $\mathrm{Sb}$ diffusion is observed in these samples before the Ga has become completely intermixed. If the sample was grown Ga-rich, it is possible that it takes a long time for the crystal to come into equilibrium with the atmosphere, and that by this time the $\mathrm{Ga}$ is homogeneous. It is therefore believed that the $\mathrm{Sb}$ diffuses under $\mathrm{Sb}$-rich conditions in an Sb-rich ambient when equilibrium is reached. In this case, Sb selfinterstitials and $\mathrm{Ga}$ vacancies are promoted, and $\mathrm{Sb}$ diffusion can take place. Sb diffusion 
under Ga-rich ambient was not observed after a 93 hour anneal at $700^{\circ} \mathrm{C}$, and the $\mathrm{Sb}$ diffusivity reported in Table 4-1 is only an upper limit.

\subsection{Zn Diffusion}

The presence of extended defects in the samples with kink and tail profiles made them unsuitable for this diffusion study. The diffusion mechanisms developed in Section 2.3 assume a regular crystalline lattice completely free of extended defects. All samples showing kink-and-tail profiles were thus ignored for all modeling. Only those samples showing box shaped profiles were used in the determination of the diffusion mechanism.

It is worthwhile however to comment briefly on the presence of the defects and their correlation to the kink and tail profile. As discussed in Section 1.4.2, both kink-andtail profiles and extended defects have been observed in this system, but there has not been any previous correlation between the two effects. This work's observation that the deeper defect band coincides closely with the high concentration kink suggests that it could be caused directly by the defect band. The observation that the kink and tail profile was only present in the those samples with $\mathrm{Zn}$ surface concentrations above $10^{20} \mathrm{~cm}^{-3}$ would seem to set a level at which extended defects are caused during $\mathrm{Zn}$ diffusion in GaSb.

Comparing the box profiles to the profiles predicted via the modeling described in Figure 2-12 and Figure 2-13 allows a number of options to be ruled out. Careful observation of the near-surface region shows a short concave-up section on the $\mathrm{Zn}$ diffused samples, ruling out the neutral and positive vacancy charge states mediating via the dissociative mechanism as well as $G a_{I}^{2+}$ and $G a_{I}^{3+}$ mediating via the kick-out mechanism. The remaining possibilities are negative vacancies mediating via the 
dissociative mechanism or neutral or singly charged self-interstitials mediating via the kick-out mechanism. Since the crystal is so heavily p-doped by the $\mathrm{Zn}$, negative point defects will be severely suppressed, making the kick-out mechanism the most likely candidate.

In fact, accurate modeling of the box profiles is achieved within the Ga selfinterstitial controlled mode of $\mathrm{Zn}$ diffusion via the kick-out mechanism. For surface concentrations of $1-2 \times 10^{19} \mathrm{~cm}^{-3}$, very good fitting comes using a neutral Ga selfinterstitial. The complete kick-out reaction equation is shown below.

$$
Z n_{i}^{+} \Leftrightarrow Z n_{G a}^{-}+I_{G a}^{0}+2 h^{+}
$$

Equation 5-2

An example of the fitting achieved for a sample in this concentration range can be seen in Figure 5-1. 


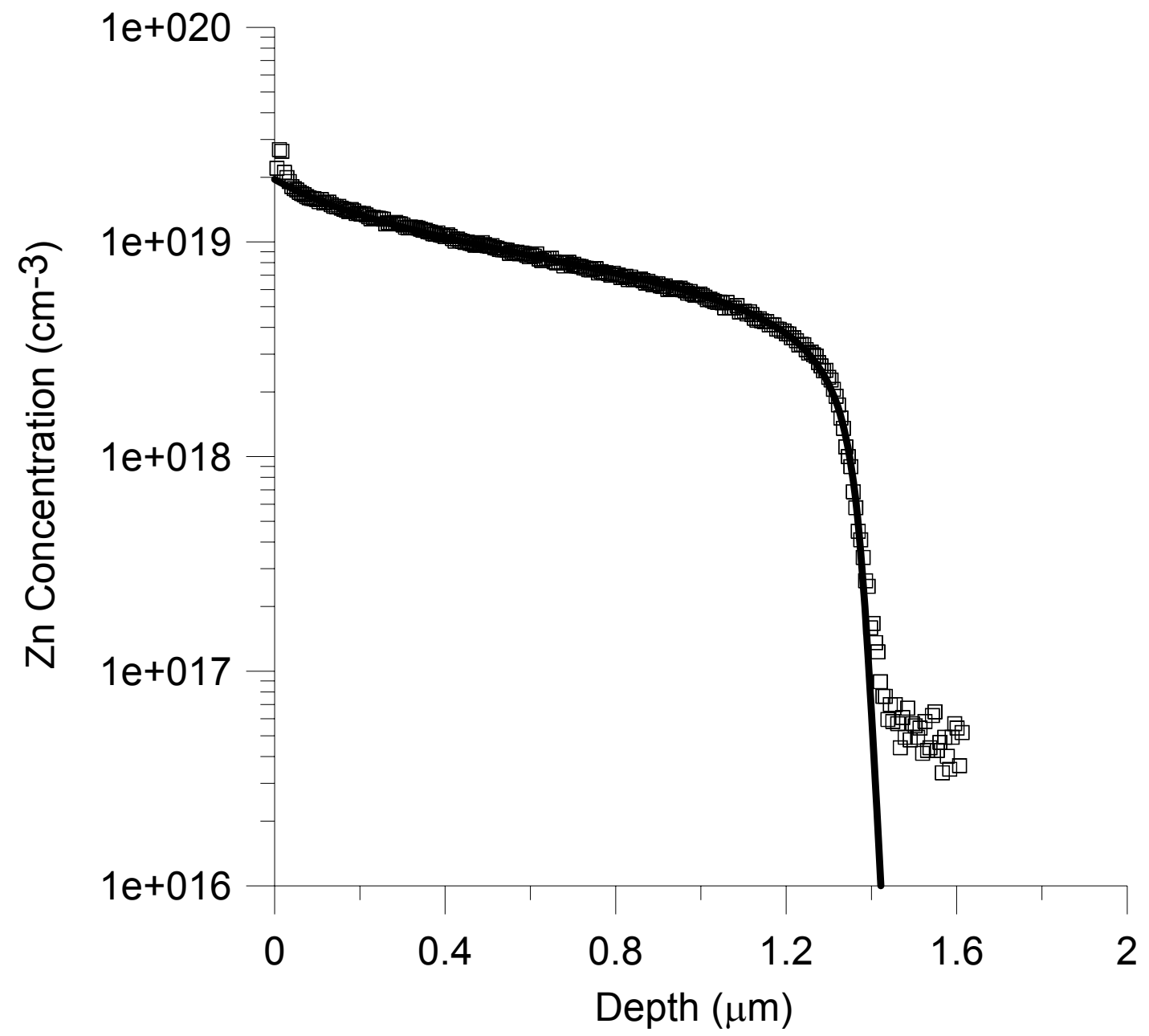

Figure 5-1: SIMS profile ( $\square$ ) and fitting (solid line) based on the neutral Ga self-interstitial controlled mode of the kick-out mechanism at a $\mathrm{Zn}$ surface concentration of approximately $2 \times 10^{19} \mathrm{~cm}^{-3}$. The sample was annealed at $609^{\circ} \mathrm{C}$ for 500 minutes using a $2 \% \mathrm{Zn} / 98 \%$ Ga source.

As the surface concentration moves above $2 \times 10^{19} \mathrm{~cm}^{-3}$, the fitting using the neutral Ga self-interstitial does not properly reproduce the data at the diffusion front. This is clearly seen in Figure 5-2 where the Zn surface concentration is $3 \times 10^{19} \mathrm{~cm}^{-3}$. We believe that in this region, the singly charged $\mathrm{Ga}$ self-interstitials begin to form compensating defects due to the extremely low $\mathrm{E}_{\mathrm{F}}$, set by the substitutional $\mathrm{Zn}$ concentration. These defects begin to mediate diffusion in addition to the neutral Ga selfinterstitial in the form of listed below. 


$$
Z n_{i}^{+} \Leftrightarrow Z n_{G a}^{-}+I_{G a}^{+}+h^{+}
$$

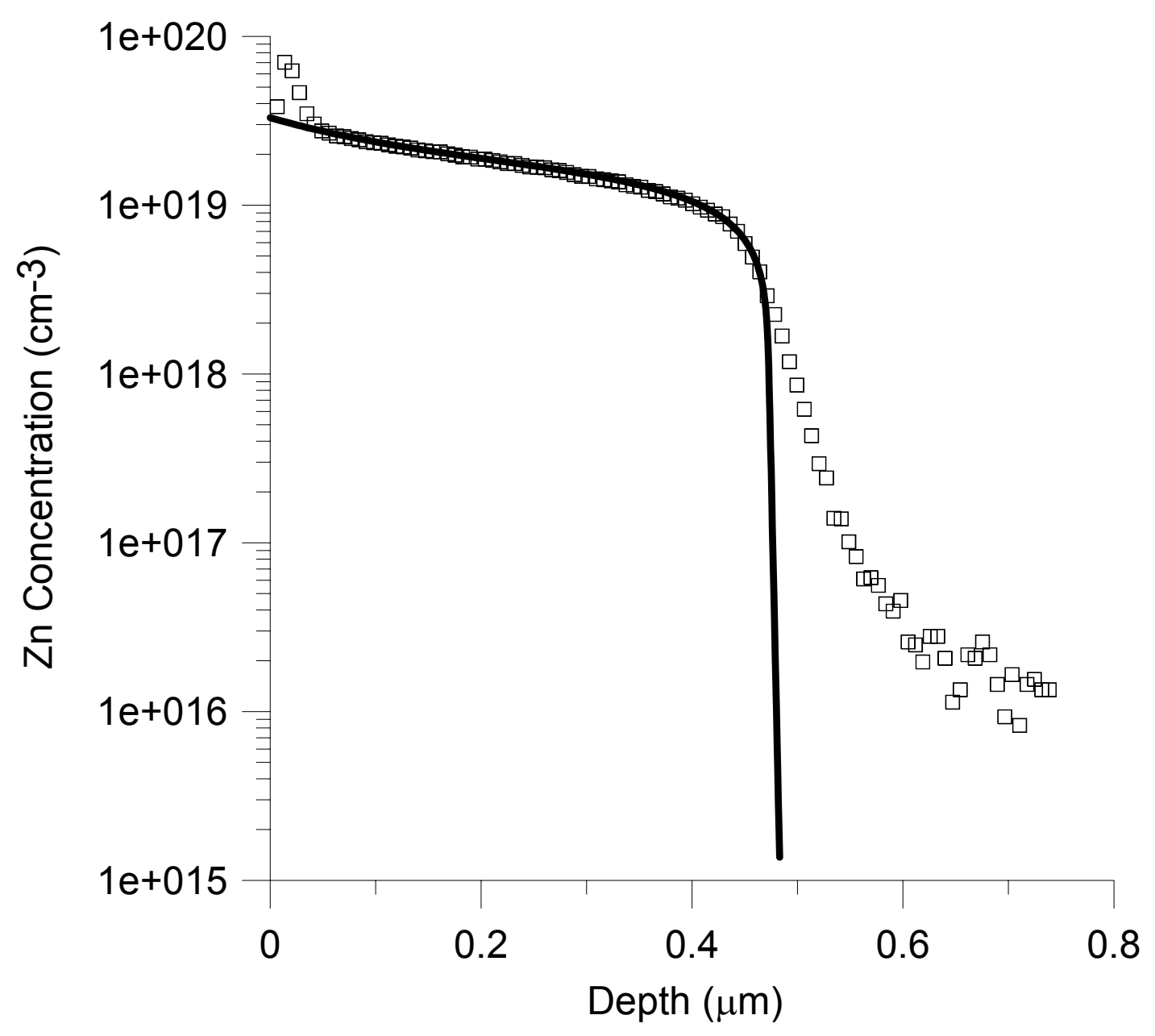

Figure 5-2: SIMS profile ( $\square$ ) and fitting (solid line) based on neutral Ga self-interstitial controlled mode of the kick-out mechanism at a $\mathrm{Zn}$ surface concentration of approximately $3 \times 10^{19} / \mathrm{cm}^{3}$. The fitting makes a clear departure from the data at the diffusion front. Sample was annealed at $561^{\circ} \mathrm{C}$ for 921 minutes using a $2 \% \mathrm{Zn} / \mathbf{9 8 \%}$ Ga source.

The finding that $\mathrm{Zn}$ diffuses in $\mathrm{GaSb}$ via the kick-out mechanism is in-line with many of the previous results. The earlier fitting of diffusion profiles indicated a substitutional-interstitial mechanism, and the kick-out is one of these. In addition to this, the TEM analysis of the extended defects caused by $\mathrm{Zn}$ diffusion with high surface concentrations indicated interstitial-type defects, a result consistent with the kick-out 
mechanism [Jäger, (2000)]. While it would not be prudent at this point to claim that diffusion at the high concentrations studied by Jäger proceeds via the same mechanism as the lower surface concentration studies discussed here, the non-conflicting evidence is a good sign supporting both findings.

The fitting of the neutral Ga self-interstitials also returns data on their contribution to Ga self-diffusion. Figure 5-3 shows the extracted contribution of $I_{G a}^{0}$ to Ga selfdiffusion overlaid with the directly measured Ga self-diffusion data (from Section 4.1). This contribution to self-diffusion equals the directly measured value within a factor of 2 . This result indicates that the neutral Ga self-interstitial very likely mediates Ga selfdiffusion under Ga-rich conditions. The singly positively charged Ga self-interstitial, however, cannot be completely excluded as a possible mediator of self-diffusion. More extensive modeling, incorporating both the neutral and singly positive charged selfinterstitial diffusion simultaneously, must be carried out to fully understand this. 


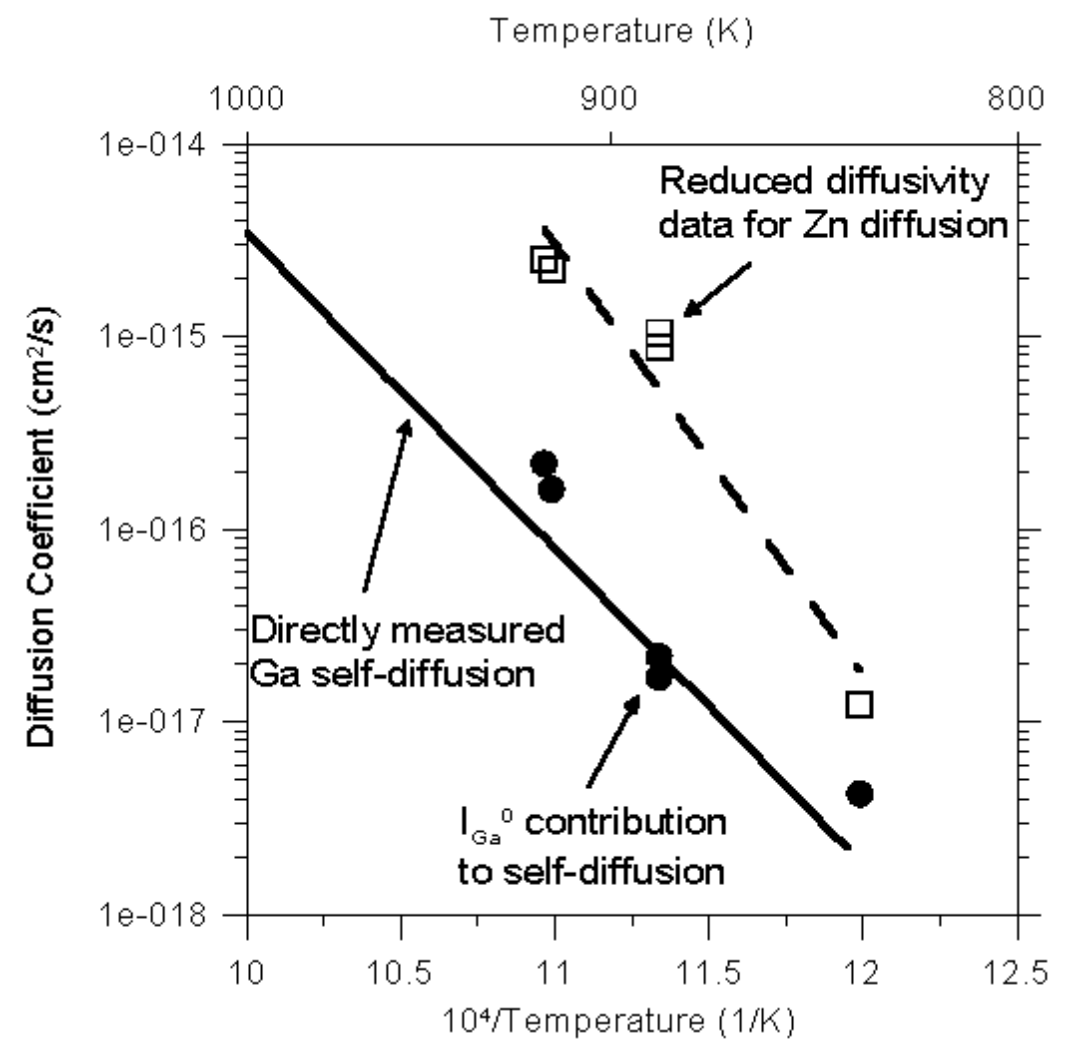

Figure 5-3: Ga and $\mathrm{Zn}$ diffusion data extracted from $\mathrm{Zn}$ diffusion profiles. Contribution of neutral Ga self-interstitial to Ga self-diffusion $(\bullet)$ as extracted from box profile diffusion profiles compared to directly measured Ga self-diffusion data (solid line). Reduced Zn diffusivity ( $\square$ ) and best fit using exponential form (dashed line).

Without the benefit of the $\mathrm{Zn}$ diffusion data, two articles were published promoting the vacancy assisted self-diffusion of Ga in GaSb [Bracht, (2000), Bracht JAP, (2001)]. An elaborate amphoteric transition model was developed, based on the $\mathrm{V}_{\mathrm{Ga}} \mathrm{Ga}$ Sb double defect, to explain how Ga vacancies could be promoted even under Ga-rich conditions. The $\mathrm{Zn}$ diffusion results provide a much more consistent picture where $\mathrm{Ga}$ self-interstitials mediate under Ga-rich conditions, as would be expected. While these results are not yet completely convincing that $\mathrm{Ga}$ diffuses exclusively via selfinterstitials, they are at least a strong indication for the self-interstitials to be involved in the diffusion process. 
The deviation from Arrhenius behavior observed in the contribution to selfdiffusion data could be explained by the normalization of the diffusivity by the $\mathrm{Zn}$ surface concentration. All the measured values for the diffusivity are dependent upon an accurate measurement of the $\mathrm{Zn}$ surface concentration. In this work, the calibration of $\mathrm{Zn}$ concentration was done using a $\mathrm{Zn}$ doped $\mathrm{CZ}$ GaSb wafer. The concentration used for the calibration $\left(10^{18} \mathrm{~cm}^{-3}\right)$ was the measured carrier concentration, but this assumes that $100 \%$ of the $\mathrm{Zn}$ is electrically active. 


\section{References}

N. Akopian, The Israel Institute of Technology, Computational Physics Web Page, http://phycomp.technion.ac.il/ nika/, (2000).

R.D. Baxter, R.T. Bate, F.J. Reid, J. Phys. Chem. Solids 26, 41 (1965).

A.W. Bett, S. Keser, O.V. Sulima, J. Crystal Growth 181, 9 (1997).

B.S. Bokshtein, S.Z. Bokshtein, A.A. Zhukhovitskii, Thermodynamics and Kinetics of Diffusion in Solids, Oxonian Press, (1985).

B.I. Boltaks, Y.A. Gutorov, Soviet Phys. Solid. St. 1, 930 (1960).

H. Bracht, E.E. Haller, K. Eberl, M. Cardona, Appl. Phys. Lett. 74, 49 (1999).

H. Bracht, S.P. Nicols, W. Walukiewicz, J.P. Silveira, F. Briones, E.E. Haller, Nature 408, 69 (2000).

H. Bracht, S.P. Nicols, E.E. Haller, J.P. Silveira, F. Briones, J. Appl. Phys. 89, 5393 (2001).

H. Bracht, Atomic Mechanisms of Mass Transport in Elelmental and Compound Semiconductors, Habilitationsschrift im Fach Physik, Universität Münster, 10 (2001).

G.J. Conibeer, A.F.W. Willoughby, C.M. Hardingham, V.K.M. Sharma, Mat. Sci. Forum 143, 1427 (1994).

G.J. Conibeer, A.F.W. Willoughby, C.M. Hardingham, V.K.M. Sharma, J. Elec. Mat. 25, 1108 (1996).

S.F. Da Cunha, J. Bougnot, Phys. Stat. Sol. A 22, 205 (1974). 
S. Dakshinamurthy, S. Shetty, I. Bhat, C. Hitchcock, R. Gutmann, G. Charache, M. Freeman, J. Electr. Mat. 28, 355 (1999).

M.W. Dvorak, C.R. Bolognesi, O.J. Pitts, S.SP. Watkins, IEEE Electron Device Letters 22, $361(2001)$.

F.H. Eisen, C.E. Birchenall, Acta Metall. 5, 265 (1957).

S. Glasstone, K. Laidler, H. Eyring, The Theory of Rate Processes; the Kinetics of $\underline{\text { Chemical Reactions, Viscosity, Diffusion and Electrochemical Phenomena, }}$ McGraw-Hill, New York and London, (1941).

G. Edelin and D. Mathiot, Philos. Mag. B 42, 95 (1980).

L.M. Fraas, W.E. Daniels, H.X. Huang, L.E. Minkin, J.E. Avery, M.J. O’Neill, A.J. McDanal, M.F. Piszczor, Submitted to 17th European Photovoltaic Solar Energy Conference, Munich, (2001).

A. Fick, Phil. Mag. and Journal of Science 10, 30 (1855).

R. Fleming, Charles Evans and Associates Website, Secondary Ion Mass Spectrometry Theory Tutorial, http://www.cea.com/cai/simstheo/caistheo.htm

F.C. Frank, D. Turnbull, Phys. Rev. 104, 617 (1956).

H.D. Fuchs, W. Walukiewicz, E.E. Haller, W. Dondl, R. Schorer, G. Abstreiter, A.I. Rudnev, A.V. Tikhomirov, V.I. Ozhogin, Phys. Rev. B 51, 16817 (1995).

U. Gösele, F. Morehead, J. Appl. Phys. 52, 4617 (1981).

E.E. Haller, J. Appl. Phys. 77, 2587 (1995).

M. Hansen, Constitution of Binary Alloys, McGraw-Hill, New York, 755 (1958). 
M. Ichimura, K. Higuchi, Y. Hattori, T. Wada, N. Kitamura, J. Appl. Phys. 68, 6153 (1990).

Ch. Jäger, W. Jäger, G. Bösker, J. Pöpping, N. Stolwijk, Phil. Mag. A 80, 1 (2000).

A.S. Kyuregyan, V.M. Stuchebnikov, Soviet Physics - Semiconductors 4, 1365 (1971).

R.F. Longini, R.F. Greene, Phys. Rev. 102, 992 (1956).

K.B. McAfee, Jr., D.M. Gay, R.S. Hozack, R.A. Laudise, G. Schwartz, W.A. Sunder, J. Cryst. Growth 76, 263 (1986).

J. Mimkes, V. Šestáková, K.M. Nassr, M. Lübbers, B. Štêpánek, J. Crystal Growth 187, 355 (1998).

S.P. Nicols, H. Bracht, M. Benamara, Z. Liliental-Weber, E.E. Haller, Physica B, Accepted for Publication July 2001

D.A. Porter, K.E. Easterling, Phase Transformations in Metals and Alloys, $2^{\text {nd }}$ Edition, Stanley Thornes, London, 43 (2000).

F.J. Reid, R.D. Baxter, S.E. Miller, J. Electrochem. Soc. 133, 713 (1966).

W. Ruhle, D. Bimberg, Phys. Rev. B 12, 2382 (1975).

E. Silveira, W. Dondl, G. Abstreiter, E.E. Haller, Phys. Rev. B 56, 2062 (1997).

M.E. Straumanis, C.D. Kim, J. Appl. Phys. 36, 3822 (1965).

V.S. Sundaram, P.E. Gruenbaum, J. Appl. Phys. 73, 3787 (1993).

L. Wang, L. Hsu, E.E. Haller, J.W. Erickson, A. Fischer, K. Eberl, M. Cardona, Phy. Rev. Lett. 76, 2342 (1996). 
L. Wang, J.A. Wolk, L. Hsu, E.E. Haller, J.W. Erickson, M. Cardona, T. Ruf, J.P. Silveira, F. Briones, Appl. Phys. Lett. 70, 1831 (1997).

G.D. Watkins, in Deep Centers in Semiconductors, A State of the Art Approach, edited by S.T. Pantelides, Gordon and Breach, New York, 147 (1986).

D. Weiler, H. Mehrer, Phil. Mag. A 49, 309 (1984). 


\section{Appendix}

\subsection{Secondary Ion Mass Spectrometry (SIMS)}

Dynamic SIMS is a powerful tool for determining elemental concentrations as a function of depth in solids. Dynamic SIMS refers to a process of etching a crater in a sample using a focused ion beam and measuring the mass to charge ratio of the backscattered ions using mass spectrometry (MS). Through careful calibration, beam focusing, and measuring only of backscattered ions from the center of the crater, an accurate plot of concentration vs. depth of multiple species can be produced.

Dr. Ron Fleming of Charles Evans and Associates developed a website with a great deal of SIMS information, and it was used as the major source for this discussion [Fleming, (2000)].

\subsubsection{Sputtering}

The most common ion beams used for SIMS analysis in semiconductors are $\mathrm{Cs}^{+}$ and $\mathrm{O}_{2}^{+}$, though $\mathrm{Ar}^{+}$, and $\mathrm{Ga}^{+}$are also occasionally used for different applications. The ion beam species is known as the primary ions. The ion beams are typically accelerated to energies ranging between 0.2 and $10 \mathrm{keV}$, depending on the depth analysis desired. For high resolution, shallow depth measurements, low energy beams are used to assure a slow sputter-etch rate, and a narrow mixing region. The beam can be focused down to below one micron in diameter, allowing for very site specific as well as lateral analyses possible.

When the primary ion beam strikes the sample surface, a number of reactions can take place. These are diagramed in Figure 7-1. First, the primary ions can become 
implanted below the sample surface. Alternatively, the impact of the primary ions can transfer energy to host matrix atoms. The region where energy from the primary ions is transferred to the matrix atoms is called the mixing zone. The mixing zone sets the fundamental limit of SIMS resolution. Some of the matrix atoms which gain energy from primary ions have velocities directed towards the surface, and can leave the solid, effectively being sputtered away. These sputtered atoms are often present in ionic forms, though there are also a large number of neutral atoms, as well as molecules. The backscattered ions are referred to as secondary ions. They can be quantified by accelerating them into a MS. The third possibility is for atoms that have gained energy from the primary ions to be lodged deeper into the material. This effect is known as knock-on. It is the most detrimental effect regarding measuring accurate depth profiles of atoms when the concentration of a species decreases rapidly, such as at an interface. A broadening of the junction is the result of atoms being "knocked" deeper in the substrate, appearing at a greater depth than from which they originated. This effect is avoided in the analysis of SIMS data by using concentration gradients that rise from low to high concentration.

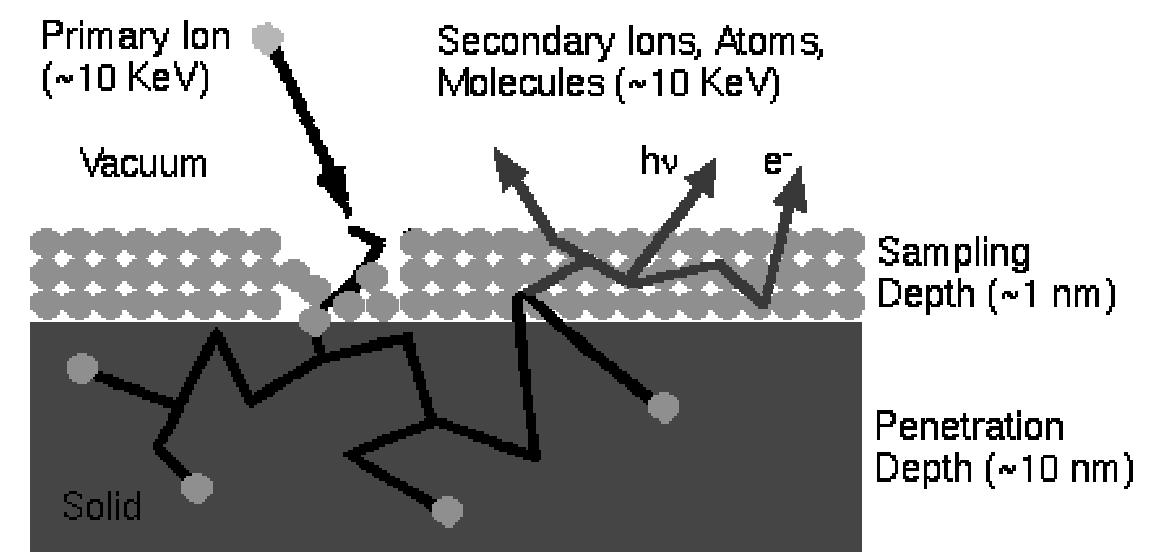

Figure 7-1: Diagram of sputtering effects in SIMS [Fleming, (2000)] 
In practice, the ion beam is scanned over a small area (typically a few hundred square microns) to produce a uniform flux of ions, resulting usually in a square crater. Equilibrium etch rates vary between 1 and $50 \AA /$ s, depending on primary beam energy and flux, crater size, sample material, as well as crystal orientation. A well tuned beam etching into a perfect crystal will maintain a flat crater bottom, but in practice imperfections in the crystal as well as in the beam lead to increasing roughness of the crater bottom as the crater deepens, resulting in lower depth resolution at larger depths.

Since the primary beam is ionic, sample charging needs to be addressed. Conducting samples are desired as the ion current can flow off to ground. In nonconducting samples, the surface can become charged, altering both the primary beam and the secondary ions. To avoid this, thin metal layers (typically gold) are often sputtered on such samples before the SIMS process is started.

\subsubsection{Detection}

In order to avoid edge effects caused by sputtering atoms from the sidewalls of the crater, apertures are used to only detect ions originating from a small area at the center of the crater.

The sputtered species which are measured to determine the concentration vs. depth plot are exclusively the secondary ions. As stated above, only a certain fraction of the sputtered species consists of ions. This fraction is referred to as the ion yield. Ion yields are specific to the element - primary ion pair as well as the matrix used. The yield of negatively charged secondary ions is increased by using a Cs beam, while the opposite is true for an $\mathrm{O}$ beam. This is the reason why detection limits for a given element vary 
with primary ion source. The periodic table below shows which beam has a higher ion yield for nearly all the elements.

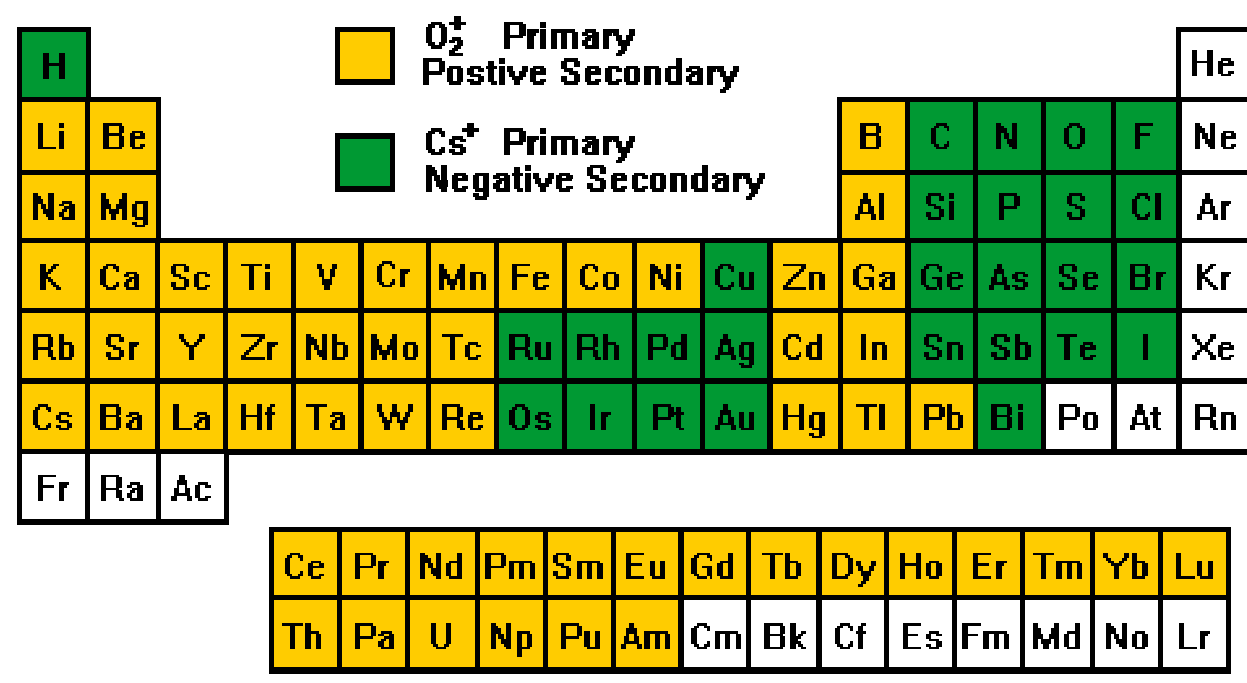

Figure 7-2: Periodic Table showing which beam has a higher ion yield for a given element [Fleming, (2000)].

The actual detection limit for each element within a given matrix can vary widely from $1 \times 10^{12}$ to $1 \times 10^{17}$ atoms $\mathrm{cm}^{-3}$. The sensitivity for an element is given by its relative sensitivity factor (RSF) within a matrix. The $R S F$ for a given element $E$ in a matrix $M$ is given by

$$
R S F=\frac{C_{E} I_{E}}{I_{M}}
$$

where $C$ is the concentration and $I$ is the intensity of the ion beam. RSF values are tabulated for common species-matrix pairs, but if no RSF is known, then a calibration standard must be used. The actual detection limit of the SIMS instrument is limited by the dark current of the detector. If the secondary ion signal is lower than that caused by stray atoms within the system and/or cosmic rays, then the element cannot be detected. 
Elements which are common in vacuum systems, such as oxygen and nitrogen have especially high detection limits.

Standards are used in order to set up an RSF table. Commonly, these standards are in the form of implants of the desired species into the desired matrix. Since ion implantation allows for accurate ion dose counting, an accurate extraction of the RSF can be made from an ion-implanted sample.

Magnetic sector or quadrupole mass spectrometers (MS) are used to actually measure the ion counts. Ions can be either atomic or molecular, but must have a mass/charge ratio lower than the maximum of the detector. It is also typical to tune the MS to fractions of a mass/charge ratio to be able to detect individual isotopes accurately. Mass interference can pose a significant problem if the species of interest has an isobaric pair. For example, ${ }^{29} \mathrm{Si}^{+}$is isobaric with $\left({ }^{1} \mathrm{H}^{28} \mathrm{Si}^{+}\right.$. High mass/charge resolution can differentiate between the two, though, and subtract out any signal if the isobaric species is known and far enough away from the species of interest. 